\title{
A high-throughput screen of inactive $X$ chromosome reactivation identifies the enhancement of DNA demethylation by 5 -aza-2'-dC upon inhibition of ribonucleotide reductase
}

\author{
Alissa Minkovsky ${ }^{1 \dagger}$, Anna Sahakyan ${ }^{1 \dagger}$, Giancarlo Bonora ${ }^{1 \dagger}$, Robert Damoiseaux ${ }^{2}$, Elizabeth Dimitrova ${ }^{3}$, \\ Liudmilla Rubbi ${ }^{4}$, Matteo Pellegrini ${ }^{4}$, Caius G. Radu ${ }^{3}$ and Kathrin Plath ${ }^{1 *}$
}

\begin{abstract}
Background: DNA methylation is important for the maintenance of the silent state of genes on the inactive $X$ chromosome (Xi). Here, we screened for siRNAs and chemicals that reactivate an Xi-linked reporter in the presence of 5-aza-2'-deoxycytidine (5-aza-2'-dC), an inhibitor of DNA methyltransferase 1, at a concentration that, on its own, is not sufficient for Xi-reactivation.

Results: We found that inhibition of ribonucleotide reductase (RNR) induced expression of the reporter. RNR inhibition potentiated the effect of 5 -aza- $2^{\prime}-\mathrm{dC}$ by enhancing its DNA incorporation, thereby decreasing DNA methylation levels genome-wide. Since both 5-aza-2'-dC and RNR-inhibitors are used in the treatment of hematological malignancies, we treated myeloid leukemia cell lines with 5-aza-2'-dC and the RNR-inhibitor hydroxyurea, and observed synergistic inhibition of cell growth and a decrease in genome-wide DNA methylation.
\end{abstract}

Conclusions: Taken together, our study identifies a drug combination that enhances DNA demethylation by altering nucleotide metabolism. This demonstrates that Xi-reactivation assays can be used to optimize the epigenetic activity of drug combinations.

Keywords: X chromosome inactivation, DNA methylation, 5-aza-2'-dC, Ribonucleotide reductase, Hydroxyurea

\section{Background}

$\mathrm{X}$ chromosome inactivation $(\mathrm{XCI})$ is a program of transcriptional gene silencing that occurs on one of two $\mathrm{X}$ chromosomes in female mammalian cells to equalize gene dosage of $\mathrm{X}$-linked genes to male cells. The inactive $\mathrm{X}$ chromosome $(\mathrm{Xi})$ is a striking example of

\footnotetext{
*Correspondence: kplath@mednet.ucla.edu

${ }^{\dagger}$ Alissa Minkovsky, Anna Sahakyan and Giancarlo Bonora contributed equally

${ }^{1}$ Department of Biological Chemistry, David Geffen School of Medicine, Eli and Edythe Broad Center for Regenerative Medicine, Jonsson Comprehensive Cancer Center, Molecular Biology Institute, University of California, 615 Charles E. Young Drive South, BSRB 390D, Los Angeles 90095, USA

Full list of author information is available at the end of the article
}

developmentally regulated heterochromatin formation in mammals. XCI has served as paradigm for understanding factors with generalized roles in gene silencing genomewide such as DNA methylation and Polycomb proteinmediated histone methylation [1-3]. The Xi is established early in female embryonic development through a series of stepwise molecular changes that cooperate to ensure stable chromosome-wide gene silencing. Once established, the $\mathrm{Xi}$ is inherited through all somatic cell divisions and adult life [1-3]. XCI is initiated by the upregulation of the long noncoding RNA Xist from the maternal or paternal X chromosome early in embryonic development [1-3]. Xist coats the X chromosome from which it is expressed and initiates a cascade of events 
including exclusion of RNA polymerase II, changes in histone marks, and recruitment of structural chromosome proteins [1-3]. Accumulation of the histone variant macroH2A1 and gain of CpG island methylation characterize the transition to the maintenance phase of XCI, which is marked by resistance to $\mathrm{X}$ chromosome reactivation (XCR) upon deletion of Xist [4-9]. Thus, Xist is absolutely required for the initiation of $\mathrm{XCI}$, but later is largely dispensable for the maintenance of the $\mathrm{Xi}$, due to the presence of various other repressive chromatin marks $[8,9]$. Notably, complete XCR is induced in vivo during pre-implantation and germ line development and in vitro by reversing cellular identity to the pluripotent state [10-13].

Despite the observation that many repressive chromatin factors are implicated in Xi establishment and maintenance, interference with DNA methylation has thus far shown the largest effect on eliciting loss of gene silencing on the $\mathrm{Xi}[5,9,14]$. It is therefore thought that DNA methylation may uniquely 'lock-in' the silenced state and execute a greater influence on the robust nature of $\mathrm{Xi}$ maintenance than other repressive regulatory mechanisms [9]. DNA methylation concentrates on CpG islands in the course of XCI with redistribution away from intragenic and intronic $\mathrm{CpGs}$ relative to the active $\mathrm{X}$ chromosome [5, 7, 15, 16, 17]. CpG island methylation on the $\mathrm{Xi}$ is established by the de novo methyltransferase DNMT3B and is subsequently propagated by the maintenance methyltransferase DNMT1 $[5,9,15]$. Interference with DNA methylation by deletion of Dnmt1 or treatment with 5-aza-2'-deoxycytidine (5-aza-2'-dC, also called decitabine) has been shown to induce the reactivation of an Xi-linked reporter gene and endogenous X-linked genes in a proportion of female somatic cells [9]. 5-aza-2'-dC is a deoxycytidine analog that upon phosphorylation incorporates into DNA and irreversibly inhibits DNMT1 [18]. Subsequent rounds of DNA replication therefore lead to passive DNA demethylation due to the absence of DNMT1 activity [19]. Together these findings indicate that $\mathrm{Xi}$ reporter systems permit the functional analysis of gene silencing, and that in addition to DNA methylation various other mechanisms contribute to Xi silencing. Therefore, $\mathrm{XCI}$ is an attractive model system to probe therapeutic approaches to the reactivation of silenced genes.

In the field of cancer biology, there is growing appreciation that abnormalities in histone modification and DNA methylation pathways can drive tumorigenesis across many cancer types and there is promise for improved therapies aimed at reversal of gene silencing [20]. In this study, we bridge the study of the $\mathrm{Xi}$ with the development of strategies to more efficiently demethylate and reactivate silenced genes. 5 -aza- $2^{\prime}-\mathrm{dC}$ is used clinically in the setting of hematologic malignancies with the rationale of reactivating silenced genes [19]. The drug is currently approved for the treatment of myelodysplastic syndrome (MDS) and acute myeloid leukemia (AML) [20]. Several studies have confirmed that 5 -aza- $2^{\prime}-\mathrm{dC}$ at low doses elicits genome-wide DNA demethylation in AML patient samples [21-23]. One approach to increase the epigenetic activity of 5-aza-2'-dC in myeloid malignancy is to use it in combination with other agents known to elicit reactivation of silenced genes, such as histone deacetylase inhibitors [20]. Notably, for the Xi, such co-treatment approaches increase the rate of XCR in cell culture systems [9]. The similar efficacy of 5-aza-2'-dC alone or in combination with other chromatin-modifying agents in Xi-linked genes and in myeloid leukemia supports the translation of findings from $\mathrm{X}$-chromosome inactivation to epigenetic cancer therapies.

Here, we set out to find additional pathways that in combination with 5-aza-2'-dC, elicit XCR. Specifically, we applied high-throughput siRNA and chemical screening to identify factors that could reactivate a silent reporter transgene that is specifically located on the Xi. Our screen employed treatment with a low dose of 5-aza-2'-dC to sensitize somatic cells for DNA demethylation and XCR, which on its own is not sufficient to induce these effects. We identified that inhibition of the ribonucleotide reductase protein complex significantly enhances the DNA demethylation action of 5-aza-2'-dC and hence the activity of the Xi-reporter. We characterize the mechanism of action as increasing DNA incorporation of 5 -aza- $2^{\prime}-\mathrm{dC}$ and thus its demethylating activity. While our approach initially centered on the $\mathrm{Xi}$, we found a pathway that altered DNA methylation levels genomewide. Our study therefore demonstrates that assays of XCR can be adapted to optimize the epigenetic activity of a DNA demethylating drug combination.

\section{Results and discussion}

An siRNA screen for XCR in the presence of a low 5-aza-2'-dC dose identifies the ribonucleotide reductase pathway

Previous work from our lab has shown that an Xi-linked, CAG promoter-driven luciferase transgene in the Hprt locus (Xi-luciferase) is a sensitive reporter of gene silencing on the $\mathrm{Xi}$ when tested in primary mouse embryonic fibroblasts (MEFs) [24]. Our Xi-luciferase MEFs faithfully inactivate the luciferase-bearing $\mathrm{X}$ chromosome in female embryonic development rather than undergoing random XCI because an Xist deletion on the other X chromosome forces XCI on the chromosome carrying the wild-type Xist allele [25] (Fig. 1a). The Xiluciferase gene body and promoter are highly methylated at the DNA level and Xi-luciferase reporter MEFs 


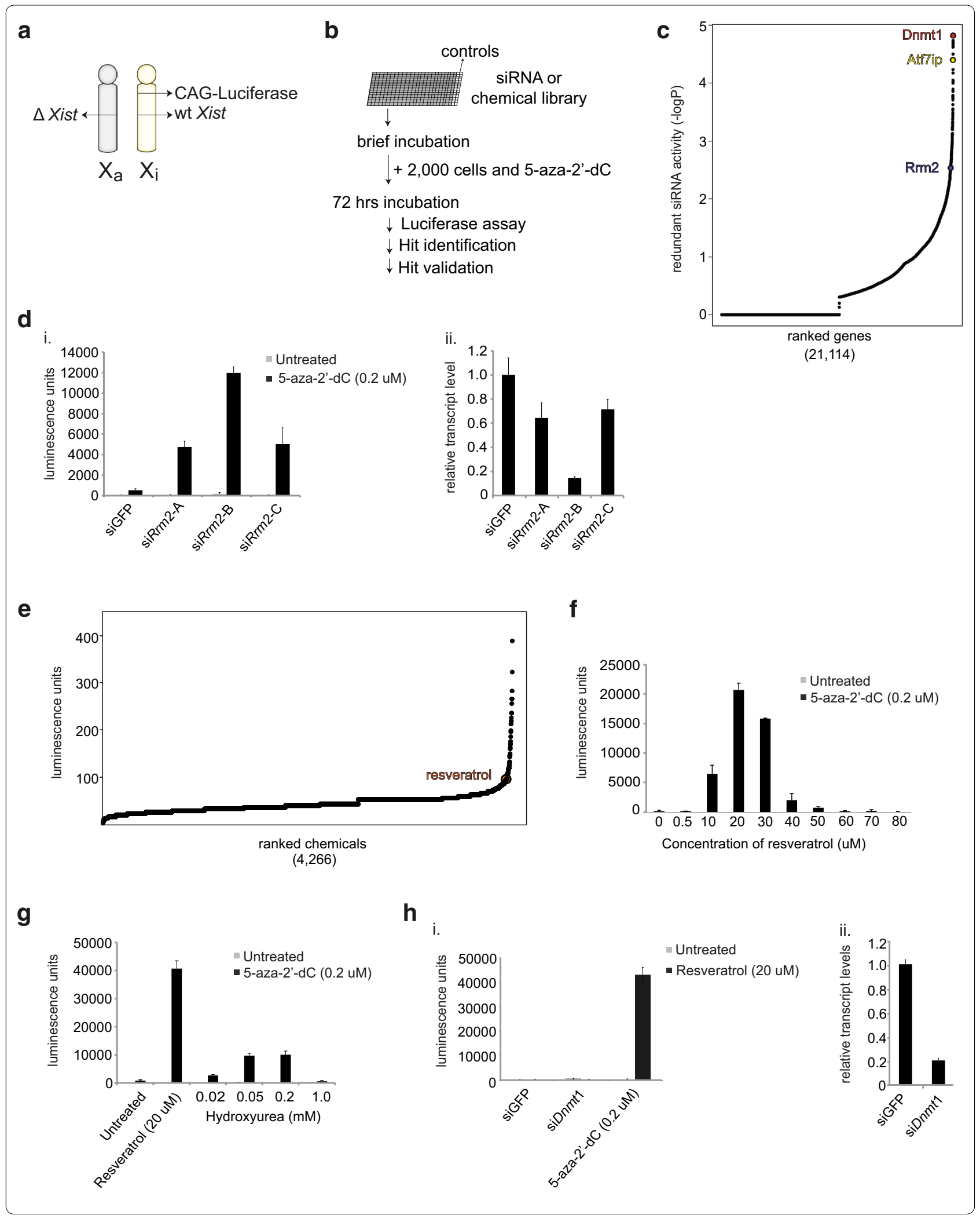


(See figure on previous page.)

Fig. 1 High-throughput siRNA and chemical screens identify RRM2 depletion and resveratrol as mediators of XCR. a Schematic of the X chromosomes in female reporter MEFs carrying the luciferase reporter transgene in the Hprt locus specifically on the Xi. The Xist deletion on one of the chromosomes skews X-inactivation to the wild-type Xist-bearing X chromosome. b Diagram of the screening workflow. siRNAs from the mouse genome-wide library and selected chemical libraries were assayed in 384-well plates containing a column of positive and negative controls. Xi-luciferase reporter MEFs were added and incubated for $72 \mathrm{~h}$ in the presence of 5-aza-2'-dC (0.2 $\mu \mathrm{M})$ prior to a luciferase assay. c Gene activity distribution plot ranked by the -log of the $p$-value obtained with the redundant siRNA activity (RSA) assay from duplicate genome-wide siRNA screens following transformation of the luminescence activity values into robust z-scores. The top validated hits, Dnmt1, Atf7ip, and Rrm2, are labeled. d ( $i$ ) Graph depicting Xi-luciferase reporter reactivation upon knockdown of $\operatorname{Rrm} 2$ with the three siRNAs $(A, B, C)$ obtained from the genome-wide library in the presence or absence of 5-aza-2'-dC $(0.2 \mu \mathrm{M})$ in the 12-well format. Luminescence was measured $72 \mathrm{~h}$ after the start of the treatment. Error bars indicate standard deviation of luminescence unit values from three individual wells with a given treatment in one experiment. (ii) RT-qPCR for RNA levels of Rrm2 normalized to siGFP control and Gapdh expression. RNA was harvested in parallel to luciferase assays shown in (i). Error bars indicate standard deviation from three measurements in one experiment. e Activity of chemicals in the chemical screen in the presence of 5-aza-2'-dC $(0.2 \mu \mathrm{M})$, ranked by luminescence unit with the value corresponding to resveratrol designated. $\mathbf{f}$ Xi-luciferase reporter assay as described in (di) titrating the resveratrol concentration with or without 5-aza-2'-dC (0.2 $\mu \mathrm{M})$. $\mathbf{g}$ Xi-luciferase reporter assay as in (di) titrating hydroxyurea (HU) with or without (untreated) 5 -aza-2'-dC $(0.2 \mu \mathrm{M})$. The result for resveratrol treatment in the same experiment is given for comparison. $\mathbf{h}$ (i) Xi-luciferase reporter assay as in (di) comparing the consequences of $0.2 \mu \mathrm{M} 5$-aza-2'-dC treatment and siRNA-mediated knockdown of Dnmt 1 to elicit reporter reactivation by $20 \mu \mathrm{M}$ resveratrol. (ii) RT-qPCR for Dnmt1 RNA levels normalized to siGFP control and Gapdh expression in the same experiment as (i)

increase luciferase activity in a dose-dependent fashion in response to 5 -aza- $2^{\prime}$-dC treatment [24]. Here, we used $\mathrm{Xi}$-luciferase reporter MEFs to screen for gene knockdowns or chemicals that could elicit XCR.

In order to perform a high-throughput screen for XCR, we established an siRNA knockdown assay in 384-well format, with each individual siRNA tested in a single well. As positive control, we chose knockdown of Dnmt1 since interference with Dnmt1 either by knockout or 5 -aza-2'-dC treatment has previously been described to elicit XCR in MEFs [9, 24]. Initially, we tested increasing concentrations of 5 -aza- $2^{\prime}-\mathrm{dC}$ in combination with Dnmt1 knockdown to determine a 5 -aza- $2^{\prime}$-dC concentration for which the depletion of Dnmt1 by siRNAs yielded robust reporter reactivation, but where 5-aza$2^{\prime}-\mathrm{dC}$ treatment alone does not elicit reactivation. Our titration experiment demonstrated that the combination of 5-aza- $2^{\prime}-\mathrm{dC}$ at a concentration ranging from 0.1 to $0.2 \mu \mathrm{M}$, along with siDnmt1 treatment, enhanced luciferase activity in the 384-well format. Importantly, 5 -aza-2'-dC treatment in this concentration range or knockdown of Dnmt1 alone did not induce a significant difference in luciferase signal compared to untreated wells (Additional file 1: Figure S1A). The requirement for 5-aza-2'-dC co-treatment with Dnmt1 knockdown to detect XCR likely reflects that Dnmt1 knockdown alone does not lead to sufficient levels of XCR detected in the small 384-well format assay. By comparison, a higher dose of 5-aza-2'-dC $(1 \mu \mathrm{M})$ elicited strong reactivation of the $\mathrm{Xi}$-linked luciferase reporter on its own that was not as dramatically enhanced by siDnmt1 treatment (Additional file 1: Figure S1A). Thus, a low dose of 5-aza$2^{\prime}-\mathrm{dC}$ has a sensitizing effect on eliciting XCR by Dnmt1 knockdown. The interaction of 5 -aza-2'-dC with other $\mathrm{Xi}$ maintenance factors indicates a similar sensitizing effect with respect to XCR. For instance, the knockdown of the candidate Xi-maintenance factor Atf7ip or deletion of Xist produces a low rate of XCR that is significantly boosted by the addition of 5 -aza-2'- $\mathrm{dC}[9,24]$. Therefore, we extended the low concentration 5-aza$2^{\prime}$ - $\mathrm{dC}$ treatment $(0.2 \mu \mathrm{M})$ to the entire genome-wide siRNA screen with the rationale that knockdown of other chromatin-modifying factors may require concurrent DNA demethylation to produce strong Xi-luciferase reporter reactivation.

We performed a genome-wide mouse siRNA screen with 51,150 siRNAs against 21,114 genes on 153 384-well plates (see "Methods" section for details on the library used) in duplicate using female Xi-linked luciferase reporter MEFs in the presence of low 5-aza-2'-dC. We measured luciferase levels $72 \mathrm{~h}$ after siRNA transfection (Fig. 1b). To eliminate batch effects, we normalized luminescence data by 384-well plate, and then analyzed the data by prioritizing gene hits with multiple active siRNAs by redundant siRNA activity (RSA) analysis [26] (Fig. 1c, Additional file 2: Figure S2). Notably, Dnmt1 was the top hit in our genome-wide screen, which provided internal validation of the method (Fig. 1c). Further support came from another hit, identified as Atf7ip, which our group recently reported as a maintenance factor in XCI [24]. As with other previously described maintenance factors, we found that the Xi-luciferase signal in response to knockdown of Atf7ip was greatly increased by low 5-aza-2'-dC $(0.2 \mu \mathrm{M})$ co-treatment [24]. Identification of Atf7ip in the screen supports the strategy of 5-aza-2'-dC co-treatment to unmask functional contribution of $\mathrm{Xi}$-maintenance factors.

To select novel hits, we chose the top 54 genes from the RSA analysis with at least two unique active siRNAs inducing an increase in luciferase levels in the 384-well 
screen, omitting genes we deemed irrelevant such as those for olfactory receptors, and retested the active siRNAs sequences from the library (Additional file 3: Table S1). Several of these siRNAs showed reproducible increases in luciferase activity in the validation assay (Additional file 4: Figure S3). We decided to focus on Rrm2 as a hit since one siRNA against it had produced the next highest level of luciferase activity in the validation assay after the siRNAs targeting Atf7ip or Dnmt1. Follow-up assays with a greater number of starting cells demonstrated an increase in luciferase activity for each of our three different siRNAs against the ribonucleotide reductase (RNR) M2 subunit gene (Rrm2) (Fig. 1d). The luminescence generated with siRrm2 treatment was in proportion to individual extent of Rrm 2 knockdown, suggesting specificity of Rrm 2 targeting for the XCR effect (Fig. 1d).

As part of the RNR enzyme complex, RRM2 catalyzes the conversion of ribonucleoside $5^{\prime}$-disphosphates to their $2^{\prime}$-deoxyribonucleoside form in the rate-limiting step of de novo dNTP biosynthesis [27]. The RRM2 subunit, which was identified in this siRNA screen, is specifically upregulated at $\mathrm{S}$ phase of cell cycle and is necessary for the activity of the RNR complex [27]. Since we identified siRrm2 in combination with 5-aza-2'-dC $(0.2 \mu \mathrm{M})$ in the genome-wide screen, we next asked if knockdown of Rrm2 could also elicit XCR in the absence of 5-aza$2^{\prime}$-dC, since interference with Atf7ip or Xist produces a low rate of XCR that is significantly boosted by the addition of 5-aza-2'-dC $[9,24]$. However, unlike previously described Xi-maintenance factors, we did not find that siRrm 2 produced luciferase activity in the absence of 5 -aza- $2^{\prime}$-dC (Fig. 1d). These results were reproduced with an Xi-linked fluorescent reporter (Additional file 5: Figure S4B/C) [9]. We conclude that low doses of 5-aza$2^{\prime}-\mathrm{dC}$ are necessary for the XCR effect of siRrm2 identified by our genome-wide screen for factors involved in the maintenance of Xi silencing.

\section{Chemical inhibitors of ribonucleotide reductase elicit XCR in the presence of 5-aza-2'-dC}

We used a complimentary approach to further probe the pathways contributing to Xi maintenance by performing a companion screen analogous to the siRNA screen but instead using a collection of annotated chemicals (Fig. 1b, Additional file 6: Figure S5A). In the screen, we found that resveratrol, a chemical agent known for mimicking cellular effects of caloric restriction, demonstrated the potential to activate the Xi-luciferase reporter (Fig. 1e) [28]. To validate the screening result, we tested the effect of various resveratrol concentrations on the Xi-luciferase reporter. The bell-shaped dose-response activity of resveratrol in combination with fixed, low concentration of
5-aza-2'-dC $(0.2 \mu \mathrm{M})$ indicated a maximal XCR activity at a concentration of $20 \mu \mathrm{M}$ (Fig. 1f). In order to confirm an XCR-specific effect, we tested whether the combination of resveratrol with 5 -aza- $2^{\prime}-\mathrm{dC}$ could reactivate different Xi-linked reporters. We found that $20 \mu \mathrm{M}$ resveratrol and 5 -aza- $2^{\prime}-\mathrm{dC}(0.2 \mu \mathrm{M})$ together could also reactivate two fluorescent reporters including the CAGdriven $\mathrm{H} 2 \mathrm{~B}$ citrine transgene within the Hprt locus and a distal CAG-driven GFP transgene (Additional file 6: Figure S4) [9, 24]. As previously observed, the proportion of cells expressing the reporter differs for Xi-CAG-H2Bcitrine and Xi-GFP MEFs likely owing to different silencing requirements of the two loci on the $\mathrm{Xi}$ [24].

Resveratrol is a naturally-occurring polyphenolic molecule believed to have numerous direct intracellular protein targets [29]. It is described to mediate its metabolic effects through direct and indirect activation of the histone deacetylase SIRT1 though no specific role in reversal of chromatin silencing or effects on the $\mathrm{Xi}$ has been described [30-32]. Of note, we did not find that knockdown of Sirt1 attenuated the ability of resveratrol with 5 -aza- $2^{\prime}-\mathrm{dC}$ to elicit Xi-luciferase reactivation (not shown). A further search for the cellular target of resveratrol in XCR led us to a study that described resveratrol as an inhibitor of RNR, the same enzyme complex that we identified as a hit in the genome-wide siRNA screen for XCR described above [33]. This link between our complimentary screening approaches pointed to resveratrol's role in XCR in the presence of 5 -aza- $2^{\prime}-\mathrm{dC}$ by means of RNR inhibition.

In order to further investigate whether RNR is the target of resveratrol in eliciting XCR, we tested a wellcharacterized inhibitor of RNR, hydroxyurea (HU), and found that it also increased Xi-luciferase activity in the presence of a low dose of 5 -aza- $2^{\prime}-\mathrm{dC}(0.2 \mu \mathrm{M})$ (Fig. $1 \mathrm{~g}$ ). From the titration, HU had a maximum effect on Xi-luciferase reactivation at 50 and at $200 \mu \mathrm{M}$ and a fading effect at $20 \mu \mathrm{M}$ (Fig. 1g). HU was not detected from the chemical library because it was assayed at a screening concentration of $10 \mu \mathrm{M}$, which was probably insufficient concentration to detect activity. $50 \mu \mathrm{M} \mathrm{HU}$ treatment in combination with low 5 -aza- $2^{\prime}-\mathrm{dC}$ also induced reactivation of the Xi-linked GFP (Additional file 5: Figure $S 4 B / C$ ). We reasoned that if resveratrol and HU converge on inhibition of RNR, that the XCR effect of resveratrol and HU should require the co-treatment with 5-aza- $2^{\prime}-\mathrm{dC}$ as seen for the Rrm 2 knockdown. Indeed, we found that similar to the siRrm2 condition, resveratrol and $\mathrm{HU}$ treatment demonstrated a complete dependence on low levels of 5 -aza-2'-dC to elicit XCR (Fig. 1d/f/g, Additional file 5: Figure S4B/C). We conclude that RNR inhibition alone by these various means does not increase Xi-luciferase activity. 
Since the Xi-luciferase reporter assay is not reflective of cell number, we measured protein concentration in luciferase assay lysates to rule out variable cell number due to different treatments as an explanation for lack of Xi-reporter activation in the absence of 5-aza-2'-dC (Additional file 7: Figure S6A). Variations in protein lysate concentration were minor across all treatments, indicating RNR inhibition requires the presence of 5 -aza-2'-dC to elicit its effect on XCR. The identification of chemicals with RNR-inhibiting activity added support to the XCR role of inhibiting this pathway in the presence of 5-aza-2'-dC.

We further investigated the relationship between 5 -aza-2'-dC and RNR inhibition in XCR by querying whether 5 -aza- $2^{\prime}-\mathrm{dC}$ can be replaced by knockdown of Dnmt1. In previous studies where 5-aza-2'-dC had a sensitizing effect towards XCR, the effect is attributable to interference with Dnmt1 [24]. For instance, 5 -aza-2'-dC treatment could be substituted by knockdown of Dnmt1 to elicit synergistic XCR by Atf7ip knockdown [24]. Contrary to these prior findings, we found that Dnmt1 depletion by siRNAs did not replace the contribution of 5 -aza-2'-dC to XCR induced by RNR-inhibition via resveratrol (Fig. 1h). Together, these findings suggest a mechanism of action whereby RNR inhibition specifically affects the action of cytidine analog 5-aza-2'-dC.

\section{RNR inhibition increases incorporation of 5-aza-2'-dC into DNA}

Next, we sought to understand how RRM2 inhibition interacts with low amounts of 5 -aza- $2^{\prime}-\mathrm{dC}$ to elicit XCR. The pool of dNTPs in the nucleus is tightly regulated and studies have speculated that RNR inhibition can increase the likelihood of nucleoside analog DNA incorporation by reducing the pools of endogenous nucleotide concentrations [27, 34]. Accordingly, we postulated that RRM2 inhibition may increase 5 -aza- $2^{\prime}$-dCTP concentration in the nucleus relative to the endogenous dCTP pool, leading to more 5-aza-2'-dCTP DNA incorporation (Fig. 2a). Higher rates of 5-aza- $2^{\prime}$ - $\mathrm{dC}$ incorporation into DNA subsequently could lead to greater DNA demethylation and XCR (Fig. 2a).

Consistent with this model, we observed that knockdown of $R r m 2$ or resveratrol treatment reproducibly increased the amount of tritiated 5-aza-2'-dC incorporated into DNA approximately by two-fold (Fig. 2b). We further tested the role of the ratio of 5 -aza- $2^{\prime}$-dCTP to endogenous dCTP by the converse manipulation of increasing dCTP relative to 5 -aza- $2^{\prime}-\mathrm{dC}$. This experiment was performed by adding increasing concentrations of deoxycytidine $(\mathrm{dC})$ into media, which is metabolized to $\mathrm{dCTP}$ within the cell, in the presence of 5 -aza- $2^{\prime}-\mathrm{dC}$ with resveratrol treatment (Fig. 2c) or $\operatorname{Rrm} 2$ knockdown
(Fig. 2d). Importantly, dC does not require the action of RNR for DNA incorporation. Our expectation was that an increase in dCTP levels in the cell would reduce the incorporation of 5 -aza- $2^{\prime}-\mathrm{dC}$ into the DNA, and therefore reduce the reactivation of the $\mathrm{Xi}$-linked luciferase reporter. As expected, the luciferase signal decreased in a dose-dependent fashion when exogenous deoxycytidine was supplied in the media (Fig. 2c/d). The loss of the Xireporter reactivation is consistent with the notion that the relative nuclear concentration of 5 -aza- $2^{\prime}-\mathrm{dCTP}$ to $\mathrm{dCTP}$ is shifted by the addition of an exogenous nucleotide substrate to reduce the effective concentration of the 5-aza-2'-dC analog (Fig. 2c/d).

An alternate explanation for the observed decrease in luciferase signal upon addition of $\mathrm{dC}$ is a reduction in cell number. To rule out possible nucleotide treatmentdependent cell growth effects, we confirmed that protein concentrations in lysates were similar for the various treatment conditions (Additional file 7: Figure S6B/C). Furthermore, we used uridine as a control because it is a nontoxic precursor of pyrimidine synthesis that, like deoxycytidine, can be taken up by cells and used as a substrate via the nucleoside salvage synthetic pathway (Fig. 2c/d) [35]. However, unlike deoxycytidine, uridine requires reduction by $\mathrm{RNR}$ in order to contribute to dNTP pools [35]. Increasing levels of uridine did not alter $\mathrm{Xi}$-luciferase levels and thereby XCR in the presence of 5-aza-2'-dC with Rrm 2 knockdown and resveratrol treatment, respectively, compared to control (Fig. 2c/d, Additional file 7: Figure $\mathrm{S6B} / \mathrm{C}$ ). These results support the role of deoxycytidine in reversing the XCR effect downstream of RNR.

In summary, RRM2/RNR inhibition was identified in the XCR screen because it augmented 5-aza- $2^{\prime}$-dC DNA incorporation. This mechanism is consistent with the observation that RNR inhibition alone, i.e. in the absence of 5-aza-2'-dC, did not produce measurable Xi-reporter reactivation in prior assays.

\section{RRM2 inhibition enhances genome-wide demethylation caused by 5 -aza-2'-dC in MEFs}

If RRM2 inhibition potentiates low dose 5-aza- $2^{\prime}-\mathrm{dC}$ action to increase XCR by increasing the incorporation of 5-aza- $2^{\prime}-\mathrm{dC}$, then DNA methylation levels in cells treated with a low dose of 5 -aza- $2^{\prime}-\mathrm{dC}$ with RRM2 inhibition should approximate those of cells treated with a high dose of 5-aza-2'-dC. We investigated DNA methylation patterns at genome-scale by reduced representation bisulfite sequencing (RRBS) [36]. Specifically, MEFs were treated with siRrm 2 or resveratrol alone, low or high doses of 5 -aza- $2^{\prime}-\mathrm{dC}$, and combinations of siRrm 2 or resveratrol with a low dose of 5-aza-2'-dC (Fig. 3, Additional file 8: Figure S7, Additional file 9: Figure S8). 


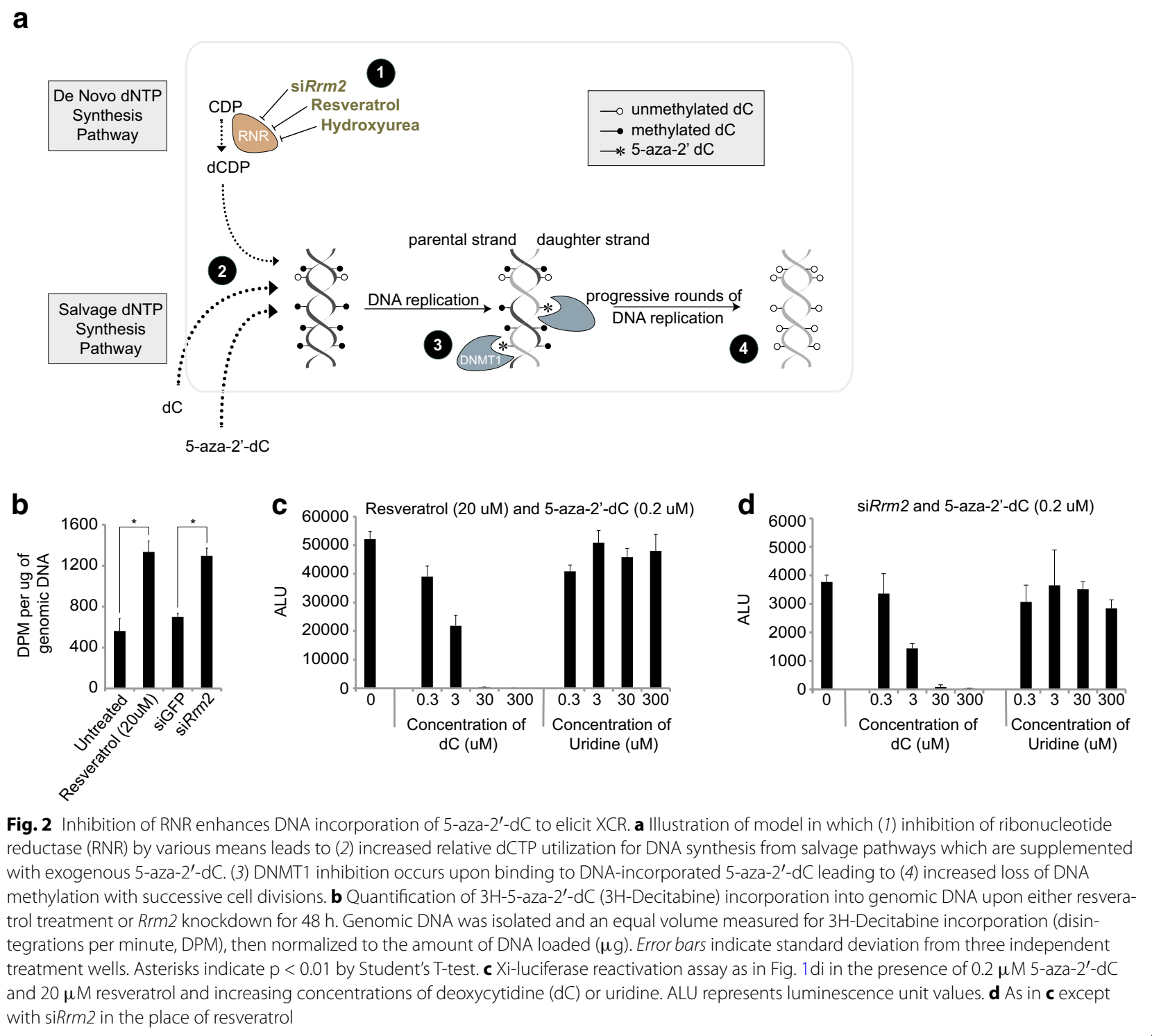

As expected, based on global methylation averages, hierarchical clustering, and methylation distributions, the treatment of MEFs with a low dose of 5-aza- $2^{\prime}-\mathrm{dC}$ $(0.2 \mu \mathrm{M})$ induced a smaller reduction in the level of genome-wide methylation than the high dose of 5-aza$2^{\prime}$-dC $(10.0 \mu \mathrm{M})$, which resulted in marked demethylation compared to control samples (Fig. 3a, b, d, Additional file 8: Figure S7C/D). We found that treatment with siRrm2 or resveratrol alone (without 5-aza2 '-dC) marginally increased global DNA methylation levels compared to untreated samples (Fig. 3a, b, d, Additional file 8: Figure S7C/D). Notably, the combination of Rrm2 knockdown or resveratrol with the low dose 5-aza- $2^{\prime}-\mathrm{dC}$ reduced global methylation to a similar extent as the high dose 5 -aza- $2^{\prime}$-dC treatment (Fig. 3a, b, d, Additional file 8: Figure S7C/D). These effects on the methylation profile were similar for autosomes and the $\mathrm{X}$ chromosome at the global scale (Fig. 3b, Additional file 9: Figure S8A) as well as on promoters and CpG islands (Additional file 8: Fig. S7A, Additional file 9: Figure S8B). These findings are consistent with a genome-wide effect on DNA methylation rather than an Xi-specific mechanism, owing to increased DNA incorporation of 5-aza2 '-dC under RRM2 inhibition conditions. We observed that CpGs with the highest levels of methylation in the control samples showed the most dramatic 5-aza-2'-dCinduced demethylation (Fig. 3c, Additional file 8: Figure S7B). For CpGs with lower methylation levels in the untreated conditions, demethylation due to 5 -aza- $2^{\prime}-\mathrm{dC}$ incorporation is still visible but less extensive (Fig. 3c, 
a

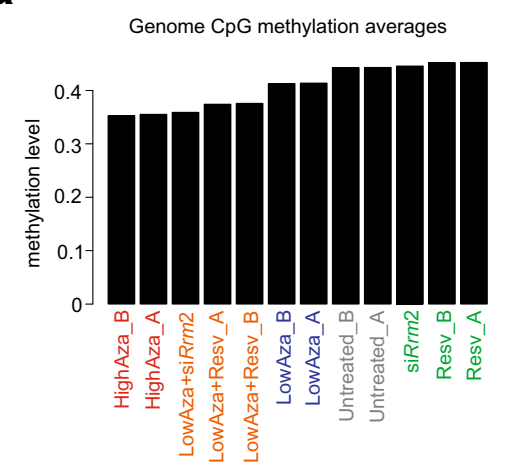

$\mathbf{C}_{\mathrm{i} .}$

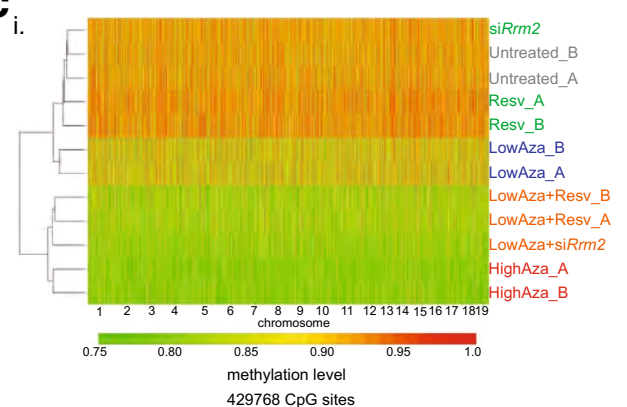

iii.

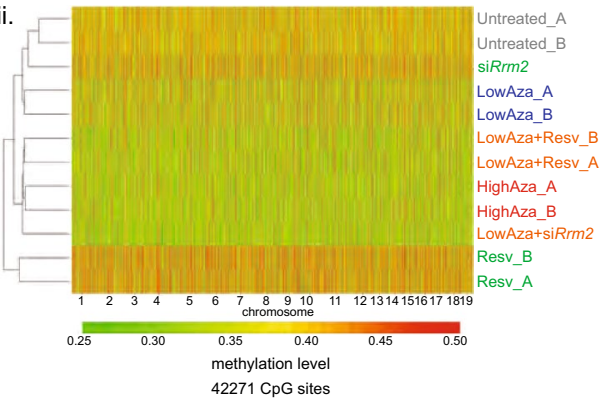

b

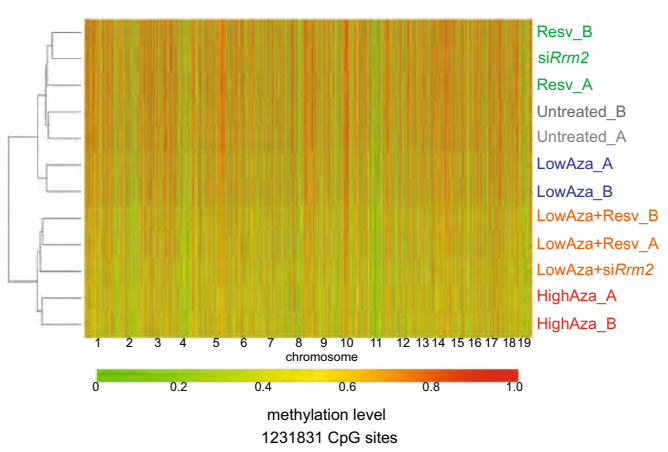

ii.

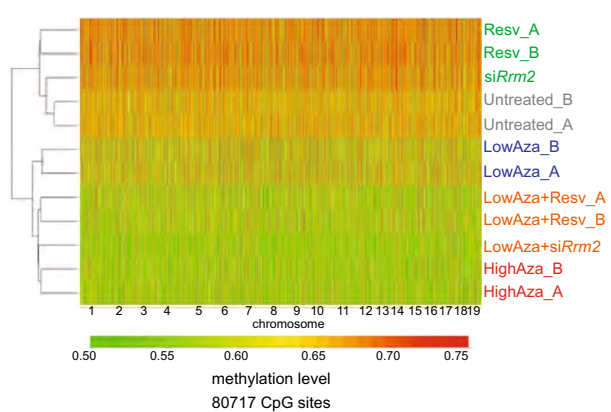

iv.

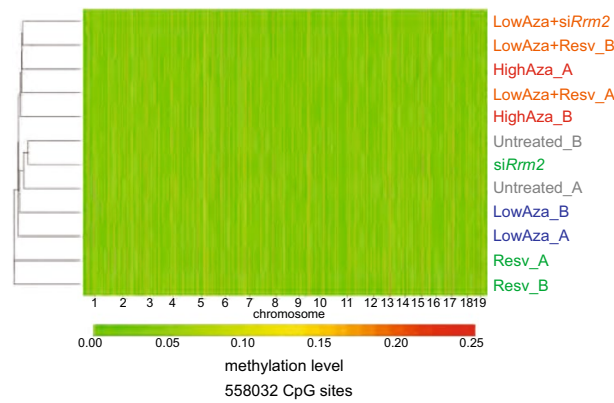

d
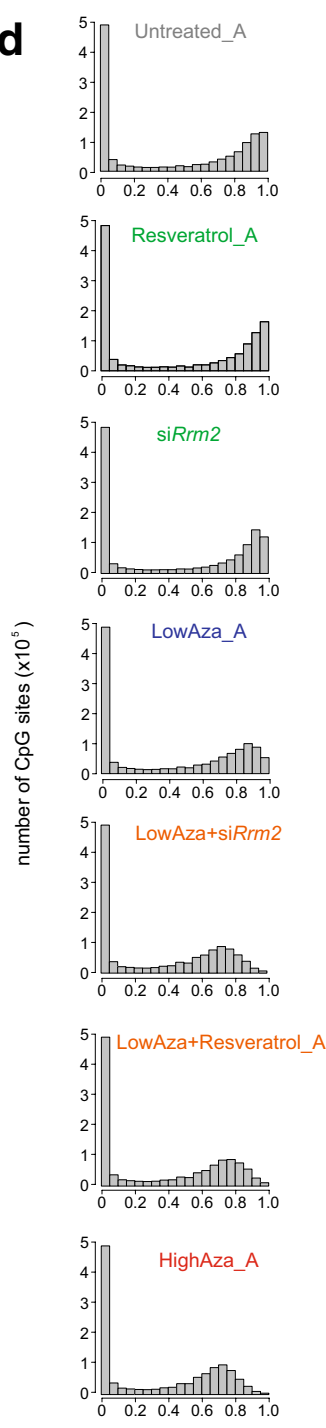

methylation level

Fig. 3 RNR inhibition increases 5-aza-2'-dC-mediated genome-wide DNA demethylation in MEFs. a Bar chart displaying average genome-wide CpG methylation levels for the indicated $72 \mathrm{~h}$ treatments filtered for CpGs with at least $5 \mathrm{X}$ sequencing coverage by RRBS across all samples. Label color reflects the various treatment groups. Subscripts ( $A$ and B) indicate replicates where applicable. Treatment concentrations are: LowAza (5-aza2'-dC $0.2 \mu \mathrm{M})$, HighAza (5-aza-2'-dC $10.0 \mu \mathrm{M}$ ), and Resv (resveratrol $20 \mu \mathrm{M})$. b Heat map of unsupervised hierarchical clustering of DNA methylation levels for all autosomal CpGs assayed by RRBS in MEFs treated with the indicated chemicals for $72 \mathrm{~h}$ as in $\mathbf{a}$ with at least $5 \mathrm{X}$ sequencing coverage across all samples. A methylation level of 1 indicates $100 \%$ methylation, while 0 represents complete absence of methylation. c Heat maps as in $\mathbf{b}$ but for subsets of autosomal $\mathrm{CpG}$ sites partitioned into four groups representing different DNA methylation levels in the untreated control samples; (i) $0.75-1.0$, (ii) $0.50-0.75$, (iii) $0.25-0.50$, and (iv) 0-0.25. In each case, the combination of RNR inhibition with $0.2 \mu M 5$-aza-2'-dC clusters away from all other samples, but together with the high dose of 5-aza-2'-dC, and is more demethylated. $\mathbf{d}$ Histograms display DNA methylation distributions for all autosomal CpGs as in $\mathbf{b}$ for indicated treatments and replicates. Data for additional replicates can be found in Additional file 8: Figure S7C/D

Additional file 8: Figure S7B). We believe that the greater apparent effect in highly methylated regions does not represent a predilection of 5 -aza- $2^{\prime}-\mathrm{dC}$ for highly methylated regions, as has been previously suggested [21], but rather that the random incorporation of 5 -aza- $2^{\prime}-\mathrm{dC}$ disproportionately affects the methylation estimates of highly methylated sites.
We also extracted the available methylation data for the $\mathrm{Xi}$-linked luciferase reporter gene to determine whether the methylation levels correlated with the extent of $\mathrm{Xi}$-luciferase reactivation in the various conditions. We found that $\mathrm{CpG}$ sites within the luciferase reporter gene followed the genome-wide methylation changes, and that the low 5 -aza- $2^{\prime}-\mathrm{dC}$ treatment 
together with RNR inhibition, by either Rrm 2 knockdown or resveratrol, induced similar demethylation as the high dose of 5 -aza-2'- $\mathrm{dC}$ (Additional file 10: Figure S9). The concordant behavior of Xi-luciferase reporter $\mathrm{CpG}$ sites supports the conclusion that the augmentation of DNA incorporation of 5 -aza- $2^{\prime}-\mathrm{dC}$ describes the $\mathrm{Rrm} 2$ result in our $\mathrm{Xi}$-reporter reactivation screen.

Taken together, our genome-wide methylation analysis for low dose 5-aza-2'-dC with RRM2 inhibition supports the idea that RRM2 inhibition increases the effective concentration of 5 -aza-2'-dC and thereby its DNA incorporation, leading to global DNA demethylation.

\section{Hydroxyurea and 5-aza-2'-dC synergistically inhibit myeloid leukemia cell line proliferation in a dose-dependent fashion}

Given that RRM2 inhibition increases DNA incorporation of 5-aza- $2^{\prime}-\mathrm{dC}$, we next applied the combination of RRM2 inhibition and 5-aza-2'-dC to a disease model in which 5 -aza- $2^{\prime}-\mathrm{dC}$ has therapeutic relevance. 5 -aza- $2^{\prime}-\mathrm{dC}$ is an FDA-approved drug and commonly used off-label in the setting of acute myeloid leukemia (AML) [20]. Therefore, we tested the drug combination in four myeloid leukemia cell lines (THP1, U937, K562, HL60) (Fig. 4, Additional file 11: Figure S10). We hypothesized that, since RRM2 inhibition increased DNA incorporation of 5 -aza- $2^{\prime}-\mathrm{dC}$, the combination of RRM2 inhibition with 5 -aza- $2^{\prime}-\mathrm{dC}$

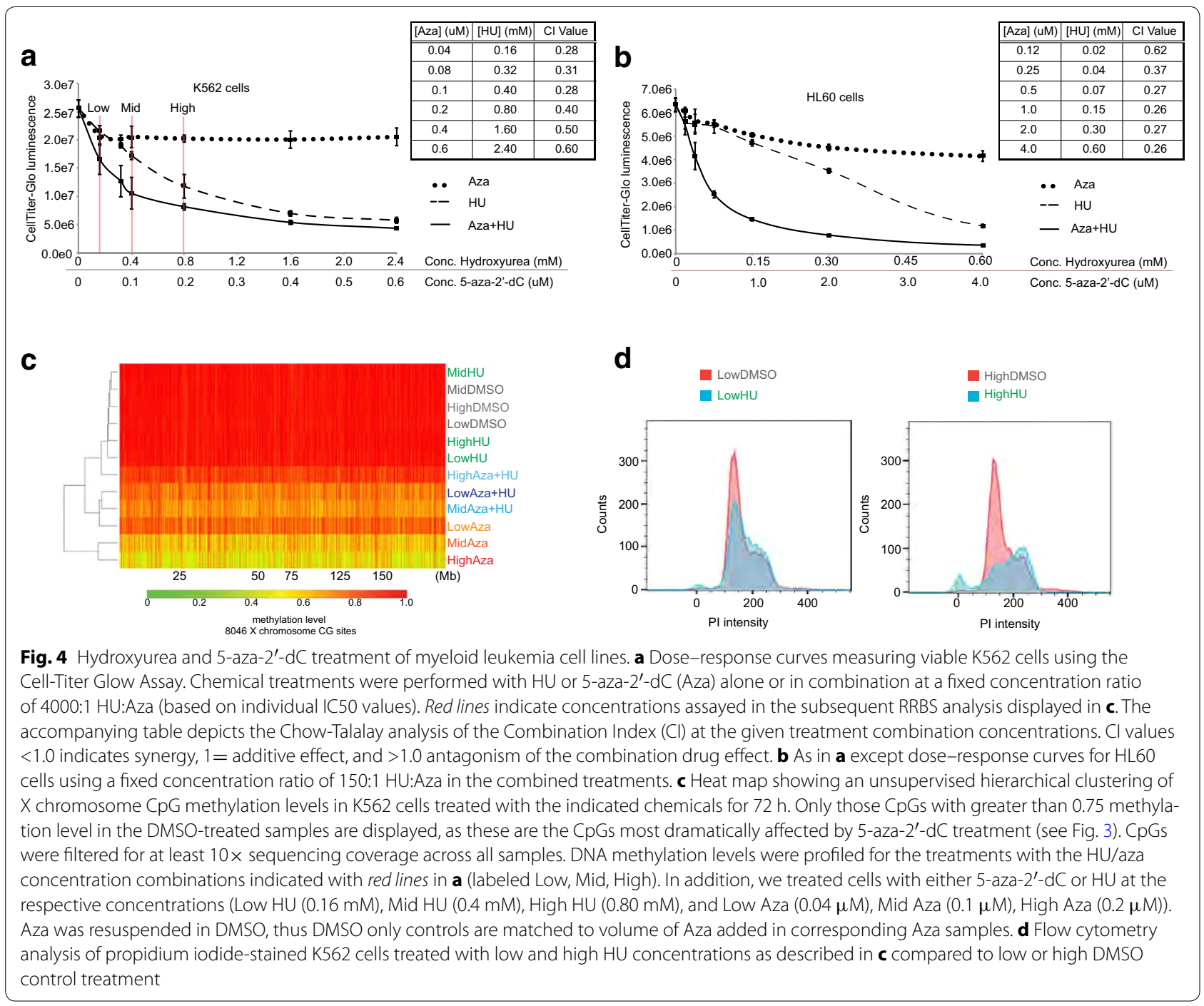


could improve the therapeutic index of 5 -aza- -2 - $d C$, allowing lower doses to maximize demethylation activity with fewer cytotoxic off-target effects. We chose to use $\mathrm{HU}$ as the form of RRM2 inhibition because it also is an FDA-approved agent commonly used off-label for cytoreductive purposes, also in the setting of AML [37].

To assess the effect of combining $\mathrm{HU}$ and 5 -aza- $2^{\prime}-\mathrm{dC}$ on myeloid leukemia cell line proliferation, we applied a luminescence-based cell viability assay that linearly scales with cell number and titered $\mathrm{HU}$ and 5-aza-2'-dC individually to determine IC50 values for each cell line (Additional file 11: Figure S10A). For 5-aza-2'-dC, IC50 values were difficult to approximate given a plateau in cell proliferation changes at higher concentrations (not shown). Thus we chose the 5-aza-2'-dC concentration corresponding to halfway to the point of plateau effect. We then combined $\mathrm{HU}$ and 5-aza-2'-dC at fixed ratios, empirically determined for each of the four myeloid leukemia cell lines (Fig. 4a/b, Additional file 11: Figure S10B/C). In each of the four cell lines tested, the combination treatment inhibited cell proliferation more than either treatment alone. In order to make a quantitative determination of the drug interaction, we calculated Chou-Talalay Combination Indices (CI) where $\mathrm{CI}<1,=1,>1$ indicate synergism, additive effect, and antagonism, respectively [38]. The combination of $\mathrm{HU}$ and 5-aza-2'-dC demonstrated evidence of drug synergism across a range of fixed drug concentration ratios in the four cell lines tested (Fig. 4a, b, Additional file 11: Figure S10B/C). We repeated the drug treatments with $\mathrm{K} 562$ cells in a soft agar assay and confirmed the synergistic effect of $\mathrm{HU}$ and 5-aza-2'-dC on clonal cell expansion (Additional file 11: Figure S10D). Consistent with the proliferation studies, the combination $\mathrm{HU}$ and 5 -aza-2' $-\mathrm{dC}$ reduced colony formation to a greater extent compared to either treatment alone. Together, these results demonstrate a synergistic interaction between $\mathrm{HU}$ and 5-aza$2^{\prime}-\mathrm{dC}$ in the control of cell proliferation.

We next assessed whether DNA demethylation related to the synergistic drug effect observed. Specifically, we determined the DNA methylation profile of K562 cells treated at a low, mid, and high concentration of 5-aza$2^{\prime}$-dC and HU at a fixed ratio by RRBS (Fig. 4c, Additional file 12: Figure S11). The low average genome-wide CpG methylation levels of approximately $35 \%$ in K562 cells with few highly methylated CpGs is consistent with a prior studies reporting overall global hypomethylation inherent to K562 cells (Additional file 12: Figure S11, DMSO-treated control conditions) [39]. Nonetheless, treatment with a fixed ratio of low $\mathrm{HU}$ and 5 -aza-2'-dC concentrations, that induced a synergistic effect on cell growth (Fig. 4a, low condition), reduced DNA methylation compared to the low 5 -aza- $2^{\prime}-\mathrm{dC}$ treatment alone (Fig. 4c). As expected, HU treatment alone did not alter
DNA methylation levels (Fig. 4c). As with MEFs, filtering by CpGs that are highly methylated in control conditions best displayed the enhancing effect of low HU to low 5-aza-2'-dC concentrations (Fig. 4c, Additional file 12: Figure S11A).

Unexpectedly, methylation levels did not appreciably decrease and even increased with the higher dose combinations of $\mathrm{HU}$ and 5-aza-2'-dC (mid and high treatment combinations) (Fig. 4c). Particularly at the high concentration combination, HU addition almost completely blunted the effect of 5 -aza-2'-dC on methylation (Fig. 4c, Additional file 12: Figure S11). We hypothesized that the differing effects of the low and high concentration combinations may be due to interference of cell cycle progression with increasing concentrations of $\mathrm{HU}$, which in turn interferes with the incorporation of 5 -aza- $2^{\prime}-\mathrm{dC}$ into DNA during DNA replication. Accordingly, flow cytometry analysis revealed a significant cell-cycle arrest of $\mathrm{K} 562$ cells at the high $\mathrm{HU}$ concentration, but not at the low concentration (Fig. 4d).

Our data suggest that at lower concentrations, HU and 5-aza-2'-dC act synergistically on cell growth, at least partially via DNA demethylation, while at higher concentrations, direct effects on cell cycle progression inhibit cell growth not allowing DNA demethylation via 5 -aza- $2^{\prime}-\mathrm{dC}$, which is replication dependent. Regardless, these data indicate that the combination of $\mathrm{HU}$ and 5 -aza- 2 '-dC synergistically decreases cell proliferation of the four myeloid leukemia cell lines tested. Moreover, the mechanism of action of this synergistic drug combination changes in a dose-dependent fashion.

\section{Conclusions}

Using an Xi-linked luciferase reporter sensitized to reactivate by low concentration 5 -aza- $2^{\prime}-\mathrm{dC}$ treatment, we screened genome-wide siRNA and chemical libraries for reactivation activity. We found that inhibition of the RRM2 subunit of the ribonucleotide reductase enzyme increases rates of Xi-linked reporter reactivation. We attribute the effect of RRM2 inhibition on the Xi in MEFs to augmentation of 5-aza-2'-dC incorporation into DNA, which in turn induces increased genome-wide DNA demethylation in a pattern similar to a high dose 5-aza-2'-dC treatment alone. Moreover, treatment of myeloid leukemia cells with 5-aza$2^{\prime}$ - $\mathrm{dC}$ and the RRM2-inhibitor HU together synergistically inhibited cell proliferation and altered DNA methylation levels in these cancer cell lines in a dose-dependent manner. These findings suggest RRM2-inhibitors improve the demethlyation activity of 5-aza-2'-dC and may have clinical benefit if used in combination.

Our screen utilized a single copy Xi-linked reporter to identify the effect of RRM2 inhibition, which was then characterized as a genome-wide effect of augmenting 
5-aza-2'-dC-mediated demethylation. The extension of our findings from a single gene reporter on the $\mathrm{Xi}$ to a genome-wide effect indicates that the $\mathrm{Xi}$ can be used as a model system for identifying and targeting general mechanisms of gene silencing. The optimal dose-schedule of 5 -aza- $2^{\prime}-\mathrm{dC}$ remains to be determined and the most effective epigenetic therapy will likely require use of 5-aza- ${ }^{\prime} \mathrm{dC}$ in combination with other epigenetic agents [40]. The XCR assay may be helpful to accomplish these objectives. The robust nature of Xi silencing in differentiated cells, however, contributes to one of the challenges of high-throughput screening with this model: XCR is partial and occurs at low rates, thus XCR assays must be optimized in sensitivity. Previous Xi maintenance screens have used pooled shRNA libraries in combination with immortalized Xi-GFP transgene-bearing reporter fibroblasts [41, 42]. The list of Xi-maintenance candidate factors from these prior studies is distinct from ours, with the exception of Dnmt1, for several potential reasons. First, previous approaches did not screen in the presence of 5-aza-2'-dC and are thus not expected to find 5-aza-2'$\mathrm{dC}$-augmenting pathways such as RRM2-inhibition. Second, cell line immortalization has the potential to create aberrancies in chromatin silencing pathways that deviate from normal development, as in cancer. Therefore, using primary MEFs, as in our screen, may more closely reflect in vivo silencing contributions of Xi maintenance pathways. However, our single-well format using individual siRNAs presents challenges in detecting rare $\mathrm{Xi}$ reactivation events, even if adapted to a more sensitive luciferase reporter gene. Our screen was likely underpowered to identify novel high-confidence Xi silencing pathways as reflected by a low signal-to-noise margin of the assay, expressed as a low Z-factor of 0.11 (Additional file 1: Figure S1B). Improvement of the assay using different co-treatments (besides 5-aza-2'-dC) to increase rates of $\mathrm{XCR}$, may lead to identification of different classes of Xi maintenance factors and minimize screening false negatives and positives [43].

Regardless, the adoption of 5 -aza-2'-dC in the optimization of this screen in order to sensitize for DNA demethylation ultimately led to identification of a 5 -aza$2^{\prime}$-dC-interacting pathway with therapeutic relevance. From the standpoint of optimizing epigenetically acting drugs, monitoring gene reactivation from the $\mathrm{Xi}$ can therefore provide a readout of chromatin reprogramming with immediate effects on gene expression.

We used cell proliferation assays and genome-wide methylation level estimates in myeloid leukemia cell lines to gauge the activity of 5 -aza- $2^{\prime}-\mathrm{dC}$. Our data suggest that at a low concentration of 5 -aza- $2^{\prime}-\mathrm{dC}$, the addition of low dose $\mathrm{HU}$, increases the fraction of 5 -aza- $2^{\prime}-\mathrm{dC}$ that is incorporated into DNA and available to inhibit DNMT1.
This DNA incorporation augmentation effect has the potential to represent a therapeutic advantage. RRM2inhibitors such as resveratrol and hydroxyurea improve the demethylation activity of 5-aza-2'-dC and may have clinical benefit if used in combination. The clinical use of 5 -aza-2'- $\mathrm{dC}$ is hampered by incomplete disease response in AML and MDS and by high rates of adverse effects $[18,44,45]$. Its mechanism of action in patients is most likely due to a combination of demethylating and direct cytotoxic actions that differ in their relative contribution according to disease context and 5-aza- $2^{\prime}-\mathrm{dC}$ concentration. At higher doses, 5 -aza- $2^{\prime}-\mathrm{dC}$ is thought to form DNA adducts leading to DNA synthesis arrest, which inhibits its DNA incorporation [20,46]. Higher doses therefore contribute to higher rates of adverse reactions including hematologic toxicities [20]. Accordingly, lower doses have been favored in more recent clinical trials and have shown greater likelihood in eliciting gene expression changes as well as producing clinic responses in AML and even solid tumors $[20,22,46]$. Thus, increasing DNA incorporation of $5-\mathrm{aza}-2^{\prime}-\mathrm{dC}$ at low doses is a promising strategy to increase its therapeutic index by biasing its activity profile towards DNA demethylation.

In this study, we observed synergistic an anti-proliferative effect of 5 -aza- $2^{\prime}-\mathrm{dC}$ in combination with HU, however, did not capture genome-wide methylation changes at all concentrations to explain this effect. The anti-proliferative effect in the absence of global DNA methylation changes is likely secondary to cytotoxic effects such as DNA adduct formation and DNA synthesis arrest. Alternatively, it is possible that differentially methylated loci are preferentially demethylated by 5 -aza- $2^{\prime}-\mathrm{dC}$ at lower concentrations and expression of these genes drives the phenotypic effects of inhibiting proliferation, even when mean global methylation levels are not affected.

Previous studies have reported that 5 -aza- $2^{\prime}-\mathrm{dC}$ and HU drug combination is antagonistic to DNA methylation based on bisulfite sequencing analysis of three loci in two other cancer cell lines [47]. Our data support these findings at high concentration $\mathrm{HU}$ with 5 -aza- $2^{\prime}-\mathrm{dC}$ in K562 cells but shows a synergistic effect on DNA demethylation at lower doses of HU and with RNR inhibition. The extent of RNR inhibition is likely critical for a synergistic interaction with $5-\mathrm{aza}-2^{\prime}-\mathrm{dC}$ as too little RNR inhibition will not increase DNA incorporation of 5-aza$2^{\prime}-\mathrm{dC}$ and too much RNR inhibition with lead to S-phase arrest and interfere with 5 -aza- $2^{\prime}-\mathrm{dC}$-mediated passive DNA demethylation (see model Fig. 2a).

Another relevant disease model to test a potential therapeutic benefit of the combination of 5-aza- $2^{\prime}-\mathrm{dC}$ and HU may be sickle cell anemia. Current therapies to treat the genetic defect in adult hemoglobin are aimed at reactivating the fetal hemoglobin gene [48]. Hydroxyurea is 
a standard therapy that when administered at cytotoxic doses to patients severely affected with sickle cell anemia increases fetal hemoglobin levels, but only in a subset of patients for unknown reasons [48]. As opposed to myeloid leukemia, where the efficacy of 5 -aza- $2^{\prime} \mathrm{dC}$ is partially attributable to demethylation, in sickle-cell anemia clinical responses to 5-aza-2'-dC do correlate with demethylation of the fetal hemoglobin gene and increases in hemoglobin levels [48-50]. Thus it is appealing to explore modified dosing schedules of $\mathrm{HU}$ and 5 -aza- 2 '- $\mathrm{dC}$ for sickle cell patients already receiving these therapies in order to potentially exploit some synergistic effect of combination therapy for raising hemoglobin levels.

\section{Methods}

\section{Genome-wide siRNA library plate preparation}

The Silencer Mouse Druggable siRNA Library V3 and Extension set V3 (Ambion) were provided as $250 \mathrm{pmol}$ of lyophilized powder in a total of 153 384-well source plates, containing one siRNA per well except in columns 23 and 24, which were reserved for controls. Each of 21,114 genes is represented by mostly 3 unique (some 2 unique) siRNAs on different 384-well plates. Plates were centrifuged at $1700 \times g, 50 \mu \mathrm{l}$ of nuclease-free water was added to each well, sealed and briefly vortexed to resuspend the siRNAs in individual wells. RNA concentrations were confirmed by measuring $1 \mu \mathrm{l}$ of siRNA solution from 14 randomly chosen wells by NanoDrop spectrophotometer (Thermo Scientific). $2 \mu$ l of siRNA diluted to $0.5 \mathrm{pmol} / \mu \mathrm{l}$ from each source plate was stamped in duplicate onto Matrix white opaque 384-well tissue culture-treated plates (Thermo Scientific) by BenchCel 4X system with a PlateLoc plate sealer, Vcode Barcode Printer, and Vprep pipette fitted with a 96 LT head (all from Agilent Technologies) and stored in $-80^{\circ}$.

\section{Derivation of MEFs}

$\mathrm{Xi}$ reporter MEFs were derived from a cross between transgenic male mice bearing a CAG promoter-driven luciferase, H2B-Citrine allele in the Hprt locus, and the $\mathrm{X}$-linked GFP, respectively, and transgenic female mice heterozygous for an Xist knockout allele [24]. MEFs were derived at embryonic day 14.5 and cultured in MEF media (DMEM supplemented with 10 \% FBS, nonessential amino acids, L-glutamine, penicillin-streptomycin, $\beta$-mercaptoethanol) following standard procedures. The reporter MEFs with genotypes $\mathrm{Xi}^{\text {CAG-Luciferase }} \mathrm{Xa}^{\Delta \text { Xist }}$, $\mathrm{Xi}^{\mathrm{CAG}-\mathrm{H} 2 \mathrm{BCitrine}} \mathrm{Xa}^{\Delta \mathrm{Xist}}$, and $\mathrm{Xi}^{\mathrm{GFP}} \mathrm{Xa}^{\Delta \text { Xist }}$ were obtained at expected Mendelian ratios of 1 out of 4 embryos and identified by PCR genotyping for presence of an Xist knockout allele, presence of a FLP-Frt recombination production in the Hprt locus and GFP, respectively, and lack of Y chromosome gene $Z f y$ [24].
High-throughput screening siRNA and chemical screening assays

The screening assay was optimized to maximize the Z-factor statistical measure of signal-to-noise ratio between the positive control of Dnmt1 knockdown and negative control or no siRNA mock-transfected cells [51]. Pilot experiments sequentially tested individual variables of the assay such as incubation times and reagent types to increase the Z-factor of the assay. The 5-aza- $2^{\prime}-\mathrm{dC}$ concentration of $0.2 \mathrm{uM}$ used in the screen was determined in this empiric fashion, by titrating a range of 5 -aza- $2^{\prime}-\mathrm{dC}$ concentrations to determine which would maximally increase the signal separation between Dnmt1 knockdown and control samples, calculated as the Z-factor of the assay. The Z-factor of the finalized screening assay was 0.11 (Additional file 1: Figure S1B) [51]. Screening data analysis was performed by first normalizing raw luminescence values by robust $\mathrm{z}$-score which is the number of median absolute deviations for a given well luminescence value from the plate median luminescence value [52].

Primary MEFs from four female Xi-luciferase reporter embryos were thawed in $15 \mathrm{~cm}^{2}$ plates, passaged twice at a 1:6 split, pooled to ensure a homogeneous cell population, and then frozen into 144 vials for use in screening and hit validation. For the large-scale screen, for each batch of 30 plates carrying the genome-wide siRNA library, 2 vials of cells were thawed in MEF media. After 1 day in culture, adherent cells were trypsinized, live cells excluding Trypan blue were counted using a hemocytometer and brought up in suspension with MEF media agitated by a stir bar.

Meanwhile, a batch of 30 plates including duplicates from 15 source plates of 384-well siRNA library were thawed at room temperature, centrifuged, and cleaned with RNAse-reducing solution (Life Technologies). A positive control siRNA targeting Dnmt1 (Ambion AM161526) was stamped by BenchCel 4X system with an 8 channel LT head (Agilent Technologies) into 16 wells of column 24 of each library plate by adding $4 \mathrm{ul}$ of nuclease-free water containing 1 pmol of siDnmt 1 to each well. The 16 wells of the column 23 were reserved as negative control and contained no siRNA. Transfection was initiated by adding $20 \mu \mathrm{l}$ of Opti-MEM (Life Technologies) and $0.05 \mu \mathrm{l}$ RNAimax (Life Technologies) per well by Multidrop 384 (Thermo Scientific) and incubating for $20 \mathrm{~min}$ to $1 \mathrm{~h} .20 \mathrm{ul}$ of cell suspension containing 2000 cells with 5 -aza- $2^{\prime}-\mathrm{dC}(0.4 \mu \mathrm{M}$, Sigma $)$ was added to the transfection mix, bringing the final 5 -aza- $2^{\prime}-\mathrm{dC}$ concentration to $0.2 \mu \mathrm{M}$. Cells were incubated for 3 days in a humidified $37^{\circ}$ incubator at $5 \% \mathrm{CO}_{2} .20 \mu$ l of media was then aspirated off using an ELx 405 plate washer (BioTek Instruments) and $20 \mu \mathrm{l}$ of One-Glo luciferase assay 
reagent (Promega) was added using the Multidrop 384 and incubated for $20 \mathrm{~min}$. As luminescence data were collected on an Acquest reader (Molecular Devices), quality control for each plate was performed by visual inspection of positive and negative controls on the heat map during data collection.

Chemical screening was performed analogously with several exceptions: 384-well plates were not pre-treated. Rather, $50 \mu \mathrm{l}$ of cell suspension with $2000 \mathrm{MEFs}$ and 5 -aza-2'-dC $(0.2 \mu \mathrm{M})$ were plated in fifteen 384-well plates. A positive control mixture was distributed to a row of wells on each plate by mixing $50 \mu \mathrm{l}$ of cell suspension with 2000 cells per well in $1 \times$ MEF media with high concentration 5-aza-2'-dC $(10.0 \mu \mathrm{M})$. The screening compounds were added to all but positive control wells as $0.5 \mu \mathrm{l}$ of $1 \mathrm{mM}$ stock in DMSO by Biomek FX (Beckman Coulter). After $72 \mathrm{~h}$ incubation, $30 \mu \mathrm{l}$ of media were aspirated off, and the luciferase assay was performed as described for the siRNA screen. Libraries screened include 4266 compounds from Microsource (2000), Biomol enzyme inhibitor (337) and bioactive lipid libraries (203), Prestwick chemical library (1120), and NIH clinical collections (606) at the UCLA MSSR [53]. The 30 chemicals producing highest luciferase values were chosen for subsequent validation.

\section{High-throughput siRNA screening analysis}

Genome-wide siRNA screen hits were identified by Redundant siRNA Activity (RSA) analysis using robust $\mathrm{z}$-scores as the input values [26]. The R script provided by Konig et al. was used with minor modifications to adapt it for our workflow (http://carrier.gnf.org/publications/ RSA). RSA works by ranking hits in order of activity then assigning $P$ values for genes based on whether their siRNAs rank higher than would be expected by chance. We obtained two activity measurements for each siRNA since the siRNA library was screened in duplicate, and treated these data points as independent measurements with regard to the analysis. Therefore, most genes were represented by six data points (and some with four data points) in the RSA analysis.

\section{Cell culture and treatment methods}

For subsequent Xi-reactivation/validation assays, MEFs at passage 1 or 2 post-derivation were seeded at a density of $6.0 \times 10^{4}$ cells per 12-well well and chemicals in MEF media and/or siRNAs in Opti-MEM media (Gibco) were added and incubated for 72 h. For 5-aza-2'-dC (Sigma), which was resuspended in DMSO and stored at $-80{ }^{\circ} \mathrm{C}$, final DMSO concentration on the cells was kept below $0.1 \%$. Total volumes of MEF and/or Opti-MEM media were normalized across samples when different treatments were used. Hydroxyurea and resveratrol (Sigma) were resuspended in DMSO and Uridine and Deoxycytidine (Sigma) were resuspended in water and stored at $-20{ }^{\circ} \mathrm{C}$. K562, HL60, U937, and THP1 cells were purchased from ATCC. K562, U937, and THP1 cells were cultured in RPMI media (Gibco) with $10 \%$ FBS and HL60 cells were cultured in IMDM (Gibco) with $20 \%$ FBS. ATCC culture method suggestions were followed for expanding the cells. The soft agar assay was performed by mixing of $1.2 \%$ nobel agar (Sigma) in water with 2X RPMI to achieve final concentration of $0.6 \%$ agar for the bottom layer. After this solidified in 6-well plates, top soft agar was prepared at final $0.3 \%$ nobel agar concentration containing $\mathrm{K} 562$ cells to achieve $4.0 \times 10^{4}$ cells per well. DMSO or 5 -aza-2' $\mathrm{dC}(0.05 \mu \mathrm{M})$ and/or HU ( $0.05 \mathrm{mM})$ were added to both bottom and top agar layers. This 1000:1 ratio of HU to 5 -aza- $2^{\prime}-\mathrm{dC}$ was determined to be optimal for the soft agar assay, which is different from the 4000:1 optimal ratio used in CellTiter Glo assay. Small colonies started appearing 4 days after plating. On day 8 , colonies were stained with $0.01 \%$ crystal violet for $1 \mathrm{~h}$, washed with $\mathrm{PBS}$, and the plates were scanned to obtain images.

\section{Luciferase assay}

For each luciferase assay, MEF Xi-luciferase reporter treatments were performed in triplicate 12-well wells for $72 \mathrm{~h}$ and lysed with $200 \mu \mathrm{l}$ passive lysis buffer (PLB, Promega) for $20 \mathrm{~min}$ at room temperature on an orbital shaker. Lysates were cleared by $30 \mathrm{~s}$ of centrifugation and $20 \mu \mathrm{l}$ were assayed for luciferase activity with $50 \mu \mathrm{l}$ of LARI reagent (Promega) on a GloMax microplate luminometer (Promega). Protein concentration measurements were performed on corresponding PLB lysates by Quick Start ${ }^{\mathrm{TM}}$ Bradford Protein Assay Kit (Bio-Rad) and analyzed by interpolating to standard curve according to the manufacturer's instruction. For the proliferation assays of leukemia cell lines, $100 \mu \mathrm{l}$ of well-suspended cells were mixed with $100 \mu \mathrm{l}$ of CellTiter $\mathrm{Glo}^{\circledR}$ reagent (Promega), incubated at room temperature for $20 \mathrm{~min}$, and luciferase units were measured using a GloMax microplate luminometer (Promega).

\section{RT-qPCR analysis}

Cells were harvested from a 6-well format in TRIzol (Invitrogen) and RNA purification was performed with the RNeasy kit (Qiagen) according to manufacturer's instructions with on-column DNAse treatment. cDNA was prepared using SuperScript III (Invitrogen) with random hexamers and RT-qPCR was performed using a $\mathrm{M} \times 3000$ thermocycler (Stratagene) with primers for Rrm2 (F-GCACTGGGAAGCTCTGAAAC, R-GGCAATTTGGAAGCCATAGA), Dnmt1 (F-CATG 
AATTCCTGCAAACAGAA, R-TTGACTTTAGCCAG GTAGCC), or Gapdh (F- GGCCTTCCGTGTTCCT, R-GCCTGCTTCACCACCTTCT). Results were normalized to Gapdh by the $\Delta \mathrm{Ct}$ method.

\section{Knockdowns in follow-up experiments}

Knockdowns by siRNA were performed by reverse transfection at $25 \mathrm{nM}$ final concentration of siRNA. Briefly, a cell suspension was added to a pre-incubated mixture of Lipofectamine RNAimax, $100 \mu \mathrm{l}$ of reduced serum OptiMEM media, and siRNA. The siRNAs used were Rrm2 [Ambion, 150659 (A), 64497 (B), 150661 (C)], Dnmt1 (Ambion, 161526), and, as negative controls, Scramble (Ambion, 4636), Luciferase (Dharmacon, D-001210-02), Aurkb (Dharmacon, D-063793-01), and GFP (Dharmacon, P-002048-01). For Rrm2 knockdown where the siRNA is not specified, siRNA 66497 was used.

\section{Flow cytometry}

Flow cytometry for measuring the reactivation of the $\mathrm{Xi}$ linked H2B Citrine and Xi-GFP reporters was performed as described previously [24]. For the cell cycle measurement with K526 cells, $5.0 \times 10^{6}$ cells (determined by trypan blue exclusion assay) were taken from each treatment condition, washed once with PBS, and stained with propidium iodide buffer ( $3 \mathrm{mM}$ EDTA $\mathrm{pH}$ 8.0, $0.05 \%$ NP40, $50 \mu \mathrm{g} / \mathrm{ml} \mathrm{PI,} 1 \mathrm{mg} / \mathrm{ml}$ RNaseA in PBS) for $30 \mathrm{~min}$ at room temperature. Stained cells were passed through a strainer and analyzed by FACSDiva (BD Biosciences) with FlowJo software (Tree Star, Inc.).

\section{$3 \mathrm{H}$ decitabine incorporation}

This assay was analogous to the reactivation treatment assays with a few modifications: assays were scaled 2.5fold to 6-well format, $1 \mu \mathrm{l}(1 \mu \mathrm{Ci})$ of tritiated 5-aza-2'-dC (3H-Decitabine, Moravek Biochemicals Inc.) was added instead of cold 5-aza-2'-dC, and samples were harvested after $48 \mathrm{~h}$ of incubation. Cells were trypsinized, genomic DNA isolated using the Quick-gDNA MinPrep kit (Zymo Research), and measured by QuBit fluorometer (Life Technologies). Tritium content of $25 \mu$ l of genomic DNA was measured using a scintillation counter and normalized to the measured DNA concentration.

\section{Reduced representation bisulfite sequencing}

Primary Xi-reporter MEFs were subjected to the same chemical treatment as used for the luciferase assays, but in 6-well format, and a fraction of the cells was taken to confirm appropriate luciferase reporter activity. Genomic DNA was isolated using the Blood and Cell Culture Mini Kit (Qiagen) with RNase A treatment (Life Technologies). The RRBS libaries were generated at previously described by Orozco et al. with minor modifications [54]. DNA purifications for each enzymatic reaction was carried out using AMPure XP beads (Beckman Coulter). Bisulfite conversion was performed using the Epitect kit (Qiagen) twice compared to manufacturer's instruction to optimize the efficiency. Bisulfite-converted libraries were amplified using MyTaq Mix (Bioline) with the following program: $\left(98{ }^{\circ} \mathrm{C}\right.$ for $15 \mathrm{~s}, 60{ }^{\circ} \mathrm{C}$ for $30 \mathrm{~s}, 72{ }^{\circ} \mathrm{C}$ for $\left.30 \mathrm{~s}\right) 12$ cycles, $72{ }^{\circ} \mathrm{C}$ for $5 \mathrm{~min}, 4{ }^{\circ} \mathrm{C}$ storage. DNA Methylation calling was performed using BS-Seeker2 (2.0.32) using Bowtie (0.12.9) for read alignment on the UCLA Hoffman2 computer cluster [55]. Reads with adapter contamination were trimmed. The adapter sequence used for the contamination check was as follows: CGAGATCGGAAGAGCACACGTC, i.e. meCG end repair $\pm \mathrm{A}$ tail $\pm 10 \mathrm{bp}$ of Illumina adapter sequence. CpG islands (CGIs) were obtained from UCSC (http:// genome.ucsc.edu) and CGI tracks were based on methods by Gardiner-Garner and Frommer [56]. Promoters were defined as the region transcription start site (TSS) minus $1 \mathrm{~kb}$ to TSS for all UCSC genes. Only sites covered by at least five reads across all samples under consideration were used in an effort to obtain reliable methylation levels. The methylation levels of samples were hierarchically clustered using complete linkage and the Euclidean distance metric. Statistical analysis, clustering, and heat map generation were performed using custom R scripts [57] (R core team, http://www.rproject.org).

\section{Public availability of data}

All genome-wide data are available from the GEO resource at http://www.ncbi.nlm.nih.gov/geo/query/acc. cgi?acc $=$ GSE72295. 


\section{Additional files}

Additional file 1: Figure S1. Optimization of 5-aza-2'-dC concentration for the genome-wide siRNA screen. A. Bar chart illustrating luciferase activity from Xi-reporter MEFs upon knockdown of Dnmt1 and treatment with varying concentrations of 5-aza-2'-dC in 384-well format for 72 hours. Error bars indicate standard deviation from eight measurements in one experiment. Aterisks indicate $p<0.01$ by Student's T-test. B. Scatterplot of luminescence values from the optimized Xi-reactivation screening assay in 384-well format in the presence of 5-aza-2'-dC (0.2 $\mu \mathrm{M})$ with siDnmt1 (red) or negative control siAurkb (Aurora kinase B, blue). The Z-factor, a measure of separation between positive and negative control populations used in the assessment of high-throughput assays, is shown [52].

Additional file 2: Figure S2. Batch effects of genome-wide siRNA screening and robust z-score normalization. A. Box plot of all raw luciferase measurements distributions per individual 384-well plate from one of the duplicates of the siRNA screen. These plates were prepared and assayed in 30-plate batches according to their numerical order in the source library plates, keeping duplicate plates together. B. As in (A) except each measurement was normalized by the robust z-score (median absolute deviations from the plate median [52].

Additional file 3: Table S3. Redundant siRNA activity ranking of genome-wide siRNA screen data. Table is color-coded to reflect which hit genes were validated.

Additional file 4: Figure S3. Validation of gene hits identified by genome-wide siRNA screening. The chart displays the luminescence for the Xi-luciferase assay in 24-well format with knockdown by the indicated siRNAs, chosen as top hits of the genome-wide screen, in combination with 5-aza-2'-dC (0.2 $\mu \mathrm{M})$ for 72 hours. For each gene hit, siRNAs were re-ordered to match the sequences of the 2 or 3 active siRNA identified by RSA activity analysis of the genome-wide siRNA screen. Error bars indicate one standard deviation from duplicate wells. siDnmt1 positive control is shown in red.

Additional file 5: Figure S4. Validation of the resveratrol result with different Xi-reporter lines. A. Diagram of MEF Xi-H2B Citrine reporter genotype. As in Fig. 1A, except the $X i$ is bearing a CAG-driven histone $\mathrm{H} 2 \mathrm{~B}-\mathrm{Citrine}$ reporter gene instead of luciferase in the Hprt locus The chart summarizes flow cytometry analysis of $\mathrm{Xi}-\mathrm{H} 2 \mathrm{~B}$ Citrine reporter MEFs treated with resveratrol $(20 \mu \mathrm{M})$ and/or 5-aza-2'-dC $(0.2 \mu \mathrm{M}$ or $10 \mu \mathrm{M})$ for 72 hours. B. Diagram of MEF Xi-GFP reporter genotype. The Xi is bearing a randomly integrated CAG-driven GFP allele near the centromere [58]. The chart summarizes flow cytometry analysis of GFP reporter MEFs treated with siRrm2, resveratrol (20 uM) or HU (0.05 mM), and DMSO or 5-aza$2^{\prime}-\mathrm{dC}(0.2 \mu \mathrm{M})$. Error bars represent standard deviation from triplicate wells. C. Representative flow cytometry dot plots of GFP reporter MEFs from part $B$.

Additional file 6: Figure S5. Chemical screen results and validation. A. Box plot of all raw luciferase measurements from the chemical screen by individual 384-well plate, demonstrating lack of obvious batch effect. Chemical library plates were prepared and assayed as one batch of 15 plates. B. Chart displaying results from the Xi-luciferase assay in the 24-well format upon treatment with various chemicals (at $10 \mu \mathrm{M}$ ) in the presence of 5-aza-2'-dC ( $0.2 \mu \mathrm{M})$ for 72 hours. Error bars indicate one standard deviation from duplicate wells except for negative control 5-aza$2^{\prime}-\mathrm{dC}(0.2 \mu \mathrm{M})$ alone $(\mathrm{n}=16)$ and positive control 5-aza-2'- $d \mathrm{C}(10.0 \mu \mathrm{M})$ alone $(n=16)$. Resveratrol is indicated with an asterisk.

Additional file 7: Figure S6. Protein concentration measurements for Xi-luciferase reactivation assays. A. Chart depicts protein concentration of cell lysates corresponding to luciferase measurements in (1G). Error bars indicate standard deviation from three individual wells. B. As in (A) but protein concentrations of cell lysates corresponding to luciferase measurements for (2C). C. As in (A) but protein concentrations of cell lysates corresponding to luciferase measurements for (2D).

Additional file 8: Figure S7. Analysis of autosomal DNA methylation in MEFs treated with combinations of RNR inhibition and 5-aza-2'-dC. A. (i) Heat map of the unsupervised hierarchical clustering as in Fig. 3B but only for autosomal $\mathrm{CpG}$ sites within $\mathrm{CpG}$ islands (CGls). Genomic locations of
CpG islands were obtained from UCSC Genome browser (see "Methods" section). Constitutively hypermethylated $(>0.75)$ and hypomethylated $(<0.15)$ sites were filtered out to improve contrast. (ii) Heat map of the unsupervised hierarchical clustering as in Fig. 3B but only for autosomal $\mathrm{CpG}$ sites within promoters. As in (i), constitutively hypermethylated $(>0.75)$ and hypomethylated $(<0.15)$ sites were filtered out to improve contrast. Promoters were defined as the region $1 \mathrm{~kb}$ upstream of the TSS for all UCSC genes. B. Heat maps of the unsupervised hierarchical clustering as in Fig. 3C for autosomal CpGs within CGls with at least 5 X coverage by RRBS across samples, but filtered for sites with methylation levels in the untreated sample of either (i) $0.75-1.0$ (ii) $0.50-.75$ (iii) $0.25-0.50$ or (iv) 0-0.25. C. As in Fig. 3D but for replicate samples. D. Pairwise significance test results conducted using a two-sample Kolmogorov-Smirnov test ('KStest stat' and 'KS-test p-value' columns) between the distributions of autosomal CpG methylation in Fig. 3D and Figure S7C, as well as two measures of effect size: Cohen's $d$ and the differences between these 'upper modes' between the comparisons ('Delta upper mode' column).

Additional file 9: Figure S8. Analysis of DNA methylation status on the $X$ chromosome in MEFs treated with combinations of RNR inhibition and 5-aza-2'-dC. A. Heat map of the unsupervised hierarchical clustering of CpG methylation levels in MEFs as in Fig. 3B, except that the data for $X$ chromosome $\mathrm{CpG}$ sites are shown. B. (i) Heat map of the unsupervised hierarchical clustering as in Fig. $3 \mathrm{~B}$ but only for $\mathrm{CpG}$ sites within CpG islands on the $\mathrm{X}$ chromosome. Constitutively hypermethylated $(>0.75)$ and hypomethylated $(<0.15)$ sites were filtered out to improve contrast. (ii) As in (i) except for CpG sites within promoters on the X chromosome. Again, constitutively hypermethylated $(>0.75)$ and hypomethylated $(<0.15)$ sites were filtered out to improve contrast. Promoters were defined as the region $1 \mathrm{~kb}$ upstream of the TSS for all UCSC genes.

Additional file 10: Figure S9. DNA methylation status of the luciferase transgene in MEFs treated with combinations of RNR inhibition and 5-aza-2'-dC. A. Bar chart displaying average CpG methylation levels for the indicated treatments filtered by CpGs with at least $5 X$ sequencing coverage by RRBS across all samples as in Fig. 3A, but only considering the CpGs in the luciferase reporter gene/promoter. B. Heat map of unsupervised hierarchical clustering of $\mathrm{CpG}$ methylation levels as in Fig. 3B except for $\mathrm{CpG}$ sites in the luciferase reporter gene/promoter. C. As in (B), except for $\mathrm{CpG}$ sites within the luciferase reporter with a methylation level greater than 0.75 in both of the untreated samples. D. Histograms showing the distribution of $\mathrm{CpG}$ methylation levels within the luciferase reporter gene.

Additional file 11: Figure S10. Synergistic effect of HU and 5-aza-2'dC on myeloid leukemia cell line proliferation. A. Graphs represent cell counts measured with the hemocytometer after trypan blue staining compared to viable cell number measurement determined by CellTiter-Glo reagent (Promega) for four myeloid leukemia cell lines. High correlation coefficient, $R^{2}$, demonstrates linear relationship. B. Dose response curves as in Fig. $4 \mathrm{~A}$, except for THP1 cells using a fixed concentration ratio of 1000:1 HU:Aza. C. Dose response curves as in Fig. 4A, except for U937 cells using a fixed concentration ratio of 300:1 HU:Aza. D. Soft agar assay of K562 cells plated in DMSO or 5-aza-2'dC (0.05 uM) and/or HU (0.05 mM) in a final concentration of $3 \%$ agar and stained with crystal violet after 8 days of growth.

Additional file 12: Figure S11. Extended data on the methylation analysis of K562 cells. A. Heat map showing an unsupervised hierarchical clustering of X chromosome $\mathrm{CpG}$ methylation in $\mathrm{K} 562$ cells treated with the indicated chemicals for 72 hours as in Fig. $4 \mathrm{C}$ but for all $\mathrm{X}$ chromosome $\mathrm{CpGs}$. B. CpG methylation distribution along the $X$ chromosome in K562 cells, for CpG sites with at least 10X coverage across all samples as determined by RRBS. Chemical treatments are as shown in Fig. 4A/C.

\section{Abbreviations}

$\mathrm{XCl}$ : $\mathrm{X}$ chromosome inactivation; $\mathrm{XCR}$ : $\mathrm{X}$ chromosome reactivation; $\mathrm{X}$ : inactive $X$ chromosome; MEFs: mouse embryonic fibroblasts; RNR: ribonucleotide reductase; HU: hydroxyurea.

\section{Authors' contributions}

AM participated in project planning, experimental data generation, data interpretation, manuscript writing, and provided some financial support. AS 
participated in project planning, experimental data generation, and data interpretation. GB performed all bioinformatics analysis. MP provided guidance for bioinformatics analysis. RD provided experimental guidance for high-throughput screening. ED participated in experimental data generation. CGR provided experimental guidance. LR participated in experimental data generation. KP conceived of the study and supervised the project, as well as participated in data interpretation, manuscript writing, and provided financial support. KP had final approval of the manuscript. All authors read and approved the final manuscript.

\section{Author details}

${ }^{1}$ Department of Biological Chemistry, David Geffen School of Medicine, Eli and Edythe Broad Center for Regenerative Medicine, Jonsson Comprehensive Cancer Center, Molecular Biology Institute, University of California, 615 Charles E. Young Drive South, BSRB 390D, Los Angeles 90095, USA. ${ }^{2}$ Department of Molecular and Medicinal Chemistry and California NanoSystems Institute, University of California, Los Angeles 90095, USA. ${ }^{3}$ Ahmanson Translational Imaging Division, Department of Molecular and Medical Pharmacology, University of California, Los Angeles 90095, USA. ${ }^{4}$ Department of Molecular, Cell, and Developmental Biology, University of California, Los Angeles 90095, USA.

\section{Acknowledgements}

K.P. is supported by the NIH (DP2OD001686 and P01 GM099134), CIRM (RN1-00564, RB3-05080, and RB4-06133), the Jonsson Comprehensive Cancer Center, and the Eli and Edythe Broad Center of Regenerative Medicine and Stem Cell Research at UCLA. A.M. is supported by National Research Service Award AG039179. K.P. and A.M. were supported for this work by funds from the Iris Cantor-UCLA Women's Health Center Executive Advisory Board. A.S. is supported by Mangasar M. Mangasarian Scholarship, UCLA Philip Whitcome pre-doctoral training fellowship, and National Research Service Award GM115122. G.B. is supported by a UCLA Dissertation Year Fellowship and the UCLA Philip Whitcome pre-doctoral training fellowship. We thank Vincent Pasque and Amy Pandya-Jones for critical reading of this manuscript. We thank Stephen Smale, Gerry Weinmaster, and H. Philip Koeffler for experimental advice, and we are grateful to Winnie Hwong for technical assistance. Funding bodies played no role in design, collection, analysis, or interpretation of data, nor in the writing of manuscript or decision to submit manuscript for publication.

\section{Competing interests}

The authors declare that they have no competing interests.

Received: 29 June 2015 Accepted: 23 September 2015 Published online: 13 October 2015

\section{References}

1. Brockdorff N. Chromosome silencing mechanisms in X-chromosome inactivation: unknown unknowns. Dev Camb Engl. 2011;138:5057-65.

2. Lee JT. Gracefully ageing at 50, X-chromosome inactivation becomes a paradigm for RNA and chromatin control. Nat Rev Mol Cell Biol. 2011;12:815-26.

3. Pollex T, Heard E. Recent advances in X-chromosome inactivation research. Curr Opin Cell Biol. 2012;24:825-32.

4. Costanzi C, Pehrson JR. Histone macroH2A1 is concentrated in the inactive X chromosome of female mammals. Nature. 1998;393:599-601.

5. Gendrel A-V, Apedaile A, Coker H, Termanis A, Zvetkova I, Godwin J, Tang YA, Huntley D, Montana G, Taylor S, Giannoulatou E, Heard E, Stancheva I, Brockdorff N. Smchd1-dependent and -independent pathways determine developmental dynamics of CpG island methylation on the inactive X chromosome. Dev Cell. 2012;23:265-79.

6. Grant M, Zuccotti M, Monk M. Methylation of CpG sites of two X-linked genes coincides with $X$-inactivation in the female mouse embryo but not in the germ line. Nat Genet. 1992;2:161-6.

7. Lock LF, Takagi N, Martin GR. Methylation of the Hprt gene on the inactive X occurs after chromosome inactivation. Cell. 1987;48:39-46.

8. Wutz A, Jaenisch R. A shift from reversible to irreversible X inactivation is triggered during ES cell differentiation. Mol Cell. 2000;5:695-705.
9. Csankovszki G, Nagy A, Jaenisch R. Synergism of Xist RNA, DNA methylation, and histone hypoacetylation in maintaining $X$ chromosome inactivation. J Cell Biol. 2001;153:773-84.

10. Mak W, Nesterova TB, de Napoles M, Appanah R, Yamanaka S, Otte AP, Brockdorff N. Reactivation of the paternal X chromosome in early mouse embryos. Science. 2004;303:666-9.

11. Okamoto I, Otte AP, Allis CD, Reinberg D, Heard E. Epigenetic dynamics of imprinted $X$ inactivation during early mouse development. Science. 2004;303:644-9.

12. Maherali N, Sridharan R, Xie W, Utikal J, Eminli S, Arnold K, Stadtfeld M, Yachechko R, Tchieu J, Jaenisch R, Plath K, Hochedlinger K. Directly reprogrammed fibroblasts show global epigenetic remodeling and widespread tissue contribution. Cell Stem Cell. 2007;1:55-70.

13. Pasque V, Tchieu J, Karnik R, Uyeda M, Sadhu Dimashkie A, Case D, Papp B, Bonora G, Patel S, Ho R, Schmidt R, McKee R, Sado T, Tada T, Meissner A, Plath K.X chromosome reactivation dynamics reveal stages of reprogramming to pluripotency. Cell. 2014;159:1681-97.

14. Blewitt ME, Gendrel A-V, Pang Z, Sparrow DB, Whitelaw N, Craig JM, Apedaile A, Hilton DJ, Dunwoodie SL, Brockdorff N, Kay GF, Whitelaw E. SmcHD1, containing a structural-maintenance-of-chromosomes hinge domain, has a critical role in X inactivation. Nat Genet. 2008;40:663-9.

15. Sado T, Fenner MH, Tan SS, Tam P, Shioda T, Li E. X inactivation in the mouse embryo deficient for Dnmt1: distinct effect of hypomethylation on imprinted and random X inactivation. Dev Biol. 2000;225:294-303.

16. Hellman A, Chess A. Gene body-specific methylation on the active X chromosome. Science. 2007;315:1141-3.

17. Weber M, Davies JJ, Wittig D, Oakeley EJ, Haase M, Lam WL, Schubeler D. Chromosome-wide and promoter-specific analyses identify sites of differential DNA methylation in normal and transformed human cells. Nat Genet. 2005;37:853-62.

18. Lyko F, Brown R. DNA methyltransferase inhibitors and the development of epigenetic cancer therapies. J Natl Cancer Inst. 2005;97:1498-506.

19. Jones PA, Taylor SM. Cellular differentiation, cytidine analogs and DNA methylation. Cell. 1980;20:85-93.

20. Azad N, Zahnow CA, Rudin CM, Baylin SB. The future of epigenetic therapy in solid tumours-lessons from the past. Nat Rev Clin Oncol. 2013;10:256-66.

21. KIco JM, Spencer DH, Lamprecht TL, Sarkaria SM, Wylie T, Magrini V, Hundal J, Walker J, Varghese N, Erdmann-Gilmore P, Lichti CF, Meyer MR, Townsend RR, Wilson RK, Mardis ER, Ley TJ. Genomic impact of transient low-dose decitabine treatment on primary AML cells. Blood. 2013;121:1633-43.

22. Tsai HC, Li H, Van Neste L, Cai Y, Robert C, Rassool FV, Shin JJ, Harbom KM, Beaty R, Pappou E, Harris J, Yen RW, Ahuja N, Brock MV, Stearns V, Feller-Kopman D, Yarmus LB, Lin YC, Welm AL, Issa JP, Minn I, Matsui W, Jang YY, Sharkis SJ, Baylin SB, Zahnow CA. Transient low doses of DNAdemethylating agents exert durable antitumor effects on hematological and epithelial tumor cells. Cancer Cell. 2012;21:430-46.

23. Yan P, Frankhouser D, Murphy M, Tam H-H, Rodriguez B, Curfman J, Trimarchi M, Geyer S, Wu Y-Z, Whitman SP, Metzeler K, Walker A, Klisovic R, Jacob S, Grever MR, Byrd JC, Bloomfield CD, Garzon R, Blum W, Caligiuri MA, Bundschuh R, Marcucci G. Genome-wide methylation profiling in decitabine-treated patients with acute myeloid leukemia. Blood. 2012;120:2466-74.

24. Minkovsky A, Sahakyan A, Rankin-Gee E, Bonora G, Patel S, Plath K. The Mbd1-Atf7ip-Setdb1 pathway contributes to the maintenance of $X$ chromosome inactivation. Epigenetics Chromatin. 2014;7:12.

25. Marahrens Y, Panning B, Dausman J, Strauss W, Jaenisch R. Xist-deficient mice are defective in dosage compensation but not spermatogenesis. Genes Dev. 1997;11:156-66.

26. Konig R, Chiang CY, Tu BP, Yan SF, DeJesus PD, Romero A, Bergauer T, Orth A, Krueger U, Zhou Y, Chanda SK. A probability-based approach for the analysis of large-scale RNAi screens. Nat Methods. 2007;4:847-9.

27. Nordlund P, Reichard P. Ribonucleotide reductases. Annu Rev Biochem. 2006;75:681-706.

28. Wood JG, Rogina B, Lavu S, Howitz K, Helfand SL, Tatar M, Sinclair D. Sirtuin activators mimic caloric restriction and delay ageing in metazoans. Nature. 2004;430:686-9. 
29. Britton RG, Kovoor C, Brown K: Direct molecular targets of resveratrol: identifying key interactions to unlock complex mechanisms. Ann NY Acad Sci 2015;1348:124-33.

30. Hubbard BP, Gomes AP, Dai H, Li J, Case AW, Considine T, Riera TV, Lee JE, SY E, Lamming DW, Pentelute BL, Schuman ER, Stevens LA, Ling AJY, Armour SM, Michan S, Zhao H, Jiang Y, Sweitzer SM, Blum CA, Disch JS, Ng PY, Howitz KT, Rolo AP, Hamuro Y, Moss J, Perni RB, Ellis JL, Vlasuk GP, Sinclair DA. Evidence for a common mechanism of SIRT1 regulation by allosteric activators. Science. 2013;339:1216-9.

31. Park S-J, Ahmad F, Philp A, Baar K, Williams T, Luo H, Ke H, Rehmann H, Taussig R, Brown AL, Kim MK, Beaven MA, Burgin AB, Manganiello V, Chung JH. Resveratrol ameliorates aging-related metabolic phenotypes by inhibiting CAMP phosphodiesterases. Cell. 2012;148:421-33.

32. Sajish M, Schimmel P. A human tRNA synthetase is a potent PARP1-activating effector target for resveratrol. Nature 2014;519:370-3.

33. Fontecave M, Lepoivre M, Elleingand E, Gerez C, Guittet O. Resveratrol, a remarkable inhibitor of ribonucleotide reductase. FEBS Lett. 1998;421:277-9.

34. Clouser CL, Chauhan J, Bess MA, van Oploo JL, Zhou D, Dimick-Gray S, Mansky LM, Patterson SE. Anti-HIV-1 activity of resveratrol derivatives and synergistic inhibition of HIV-1 by the combination of resveratrol and decitabine. Bioorg Med Chem Lett. 2012;22:6642-6.

35. Loffler M, Fairbanks LD, Zameitat E, Marinaki AM, Simmonds HA. Pyrimidine pathways in health and disease. Trends Mol Med. 2005;11:430-7.

36. Meissner A, Gnirke A, Bell GW, Ramsahoye B, Lander ES, Jaenisch R. Reduced representation bisulfite sequencing for comparative highresolution DNA methylation analysis. Nucleic Acids Res. 2005;33:5868-77.

37. Ganzel C, Becker J, Mintz PD, Lazarus HM, Rowe JM. Hyperleukocytosis, leukostasis and leukapheresis: practice management. Blood Rev. 2012;26:117-22

38. Chou T-C. Theoretical basis, experimental design, and computerized simulation of synergism and antagonism in drug combination studies. Pharmacol Rev. 2006;58:621-81.

39. Shipony Z, Mukamel Z, Cohen NM, Landan G, Chomsky E, Zeliger SR, Fried YC, Ainbinder E, Friedman N, Tanay A. Dynamic and static maintenance of epigenetic memory in pluripotent and somatic cells. Nature. 2014;513:115-9.

40. Issa JPJ, Kantarjian HM. Targeting DNA methylation. Clin Cancer Res Off J Am Assoc Cancer Res. 2009;15:3938-46.

41. Bhatnagar S, Zhu X, Ou J, Lin L, Chamberlain L, Zhu LJ, Wajapeyee N, Green MR. Genetic and pharmacological reactivation of the mammalian inactive X chromosome. Proc Natl Acad Sci USA. 2014;111:12591-8.

42. Chan KM, Zhang H, Malureanu L, van Deursen J, Zhang Z. Diverse factors are involved in maintaining $X$ chromosome inactivation. Proc Natl Acad Sci. 2011;108:16699-704.

43. Minajigi A, Froberg JE, Wei C, Sunwoo H, Kesner B, Colognori D, Lessing D, Payer B, Haas W, Lee JT. A comprehensive Xist interactome reveals cohesin repulsion and an RNA-directed chromosome conformation. Science. 2015;349:aab2276-2276.

44. Kantarjian H, Oki Y, Garcia-Manero G, Huang X, O'Brien S, Cortes J, Faderl S, Bueso-Ramos C, Ravandi F, Estrov Z, Ferrajoli A, Wierda W, Shan J, Davis J, Giles F, Saba HI. Issa J-PJ: Results of a randomized study of 3 schedules of low-dose decitabine in higher-risk myelodysplastic syndrome and chronic myelomonocytic leukemia. Blood. 2007;109:52-7.
45. Kantarjian HM, Thomas XG, Dmoszynska A, Wierzbowska A, Mazur G, Mayer J, Gau J-P, Chou W-C, Buckstein R, Cermak J, Kuo C-Y, Oriol A, Ravandi F, Faderl S, Delaunay J, Lysák D, Minden M, Arthur C. Multicenter, randomized, open-label, phase III trial of decitabine versus patient choice, with physician advice, of either supportive care or low-dose cytarabine for the treatment of older patients with newly diagnosed acute myeloid leukemia. J Clin Oncol Off J Am Soc Clin Oncol. 2012;30:2670-7.

46. Issa JP, Garcia-Manero G, Giles FJ, Mannari R, Thomas D, Faderl S, Bayar E, Lyons J, Rosenfeld CS, Cortes J, Kantarjian HM. Phase 1 study of low-dose prolonged exposure schedules of the hypomethylating agent 5-aza2'-deoxycytidine (decitabine) in hematopoietic malignancies. Blood. 2004;103:1635-40.

47. Choi SH, Byun HM, Kwan JM, Issa JP, Yang AS. Hydroxycarbamide in combination with azacitidine or decitabine is antagonistic on DNA methylation inhibition. Br J Haematol. 2007;138:616-23.

48. Saunthararajah Y, Hillery CA, Lavelle D, Molokie R, Dorn L, Bressler $L$, Gavazova S, Chen YH, Hoffman R, DeSimone J. Effects of 5-aza2'-deoxycytidine on fetal hemoglobin levels, red cell adhesion, and hematopoietic differentiation in patients with sickle cell disease. Blood. 2003;102:3865-70.

49. DeSimone J, Koshy M, Dorn L, Lavelle D, Bressler L, Molokie R, Talischy N. Maintenance of elevated fetal hemoglobin levels by decitabine during dose interval treatment of sickle cell anemia. Blood. 2002;99:3905-8.

50. Saunthararajah Y, Molokie R, Saraf S, Sidhwani S, Gowhari M, Vara S, Lavelle D, DeSimone J. Clinical effectiveness of decitabine in severe sickle cell disease. Br J Haematol. 2008;141:126-9.

51. Zhang Chung. Oldenburg: a simple statistical parameter for use in evaluation and validation of high throughput screening assays. J Biomol Screen. 1999:4:67-73.

52. Birmingham A, Selfors $L M$, Forster T, Wrobel D, Kennedy CJ, Shanks E, Santoyo-Lopez J, Dunican DJ, Long A, Kelleher D, Smith Q, Beijersbergen RL, Ghazal P, Shamu CE. Statistical methods for analysis of high-throughput RNA interference screens. Nat Methods. 2009;6:569-75.

53. Damoiseaux R. UCLA's molecular screening shared resource: enhancing small molecule discovery with functional genomics and new technology. Comb Chem High Throughput Screen. 2014;17:356-68.

54. Orozco LD, Morselli M, Rubbi L, Guo W, Go J, Shi H, Furlotte NA, Lopez D, Bennett BJ, Farber, Charles R, Ghazalpour A, Zhang MQ, Bahous R, Rozen R, Lusis AJ, Pellegrini M: Epigenome-wide association of complex metabolic traits in mice. Cell Metab. 2015;21:905-17.

55. Langmead B, Trapnell C, Pop M, Salzberg SL. Ultrafast and memoryefficient alignment of short DNA sequences to the human genome. Genome Biol. 2009;10:R25

56. Gardiner-Garden M, Frommer M. CpG Islands in vertebrate genomes. J Mol Biol. 1987;196:261-82.

57. Team RC: R: A Language and Environment for Statistical Computing Vienna, Austria, 2011. http://www.R-Proj.Org 2013.

58. Hadjantonakis AK, Cox LL, Tam PP, Nagy A. An X-linked GFP transgene reveals unexpected paternal X-chromosome activity in trophoblastic giant cells of the mouse placenta. Genes N Y N. 2000;2001(29):133-40.

\section{Submit your next manuscript to BioMed Central and take full advantage of:}

- Convenient online submission

- Thorough peer review

- No space constraints or color figure charges

- Immediate publication on acceptance

- Inclusion in PubMed, CAS, Scopus and Google Scholar

- Research which is freely available for redistribution

Submit your manuscript at

www.biomedcentral.com/submit
C Biomed Central 